\title{
ON THE DIRICHLET PROBLEM FOR SECOND-ORDER ELLIPTIC INTEGRO-DIFFERENTIAL EQUATIONS
}

\author{
G. BARLES, E. CHASSEIGNE, AND C. IMBERT
}

\begin{abstract}
In this article, we consider the analogue of the Dirichlet problem for second-order elliptic integro-differential equations, which consists in imposing the "boundary conditions" in the whole complementary of the domain. We are looking for conditions on the differential and integral parts of the equation in order to ensure that the Dirichlet boundary condition is satisfied in the classical sense or, in other words, in order that the solution agrees with the Dirichlet data on the boundary of the domain. We also provide a general existence result of a continuous viscosity solution of the non-local Dirichlet problem by using Perron's method.
\end{abstract}

Keywords: integro-differential equations, Dirichlet problem, Lévy operators, general non-local operators, viscosity solutions

Mathematics Subject Classification: 47G20, 35D99, 35J60, 35J65, 35J67, 49L25

\section{INTRODUCTION}

In this paper, we consider the analogue of the Dirichlet problem for second-order, possibly degenerate and non-linear, elliptic integro-differential equations. It is well-known that, for these non-local equations, the value of the solution has to be prescribed not only on the boundary of the domain but also in its whole complementary (see for instance [11]). Such problems have been already addressed by using different theory: we refer to Bony, Courrège and Priouret [6] for a semi-group approach and to Garroni and Menaldi [11] for a classical PDE approach using Sobolev spaces.

We consider here the viscosity solutions' approach where the Dirichlet boundary condition may be satisfied only in a generalized sense (see for example the User's guide [9]) and the main question we address is: does the solution satisfy the boundary condition in the classical sense? An almost immediate corollary of a positive answer to this question is the existence of a continuous solution for the Dirichlet problem, as we show it.

Let us be more specific now. The problems we look at can be written under the form

$$
\begin{aligned}
F\left(x, u, D u, D^{2} u, \mathcal{I}[u](x)\right) & =0 & & \text { in } \Omega, \\
u & =g & & \text { on } \Omega^{c},
\end{aligned}
$$

where $\Omega$ is a $C^{2}$-open subset of $\mathbb{R}^{N}, \Omega^{c}$ denotes its complementary and $F: \bar{\Omega} \times \mathbb{R} \times \mathbb{R}^{N} \times \mathbb{S}^{N} \times \mathbb{R} \rightarrow$ $\mathbb{R}$ is a continuous function, where $\mathbb{S}^{N}$ denotes the space of $N \times N$ symmetric matrices. We assume that $F$ satisfies the local and non-local degenerate ellipticity condition(s): for any $x \in \mathbb{R}^{N}, u \in \mathbb{R}$, $p \in \mathbb{R}^{N}, X, Y \in \mathbb{S}^{N}, l_{1}, l_{2} \in \mathbb{R}$

$$
F\left(x, u, p, X, l_{1}\right) \leq F\left(x, u, p, Y, l_{2}\right) \quad \text { if } X \geq Y, l_{1} \geq l_{2} .
$$

As we point it out by making such an assumption, the fact that $F(x, u, p, M, l)$ is non-increasing in $l$ is indeed part of the ellipticity assumption on $F$. 
Finally, concerning the non-local term, we have typically in mind operators of Lévy's type which, in $\mathbb{R}^{N}$, have the form

$$
\mathcal{I}_{L}[u](x)=\int_{\mathbb{R}^{N}}\left(u(x+z)-u(x)-\nabla u(x) \cdot z \mathbf{1}_{B}(z)\right) d \mu_{x}(z)
$$

where the $\mu_{x}$ are (a priori) singular measures with a singularity at $0, B$ is the unit ball centered at 0 and $\mathbf{1}_{B}$ denotes the indicator function of $B$. We always assume that there exists $\bar{c}>0$ such that, for any $x \in \bar{\Omega}$

$$
\int_{|z|<1}|z|^{2} d \mu_{x}(z)+\int_{|z| \geq 1} d \mu_{x}(z) \leq \bar{c}<+\infty .
$$

This assumption is natural from the probabilistic point of view; a measure satisfying (4) is referred to as a Lévy one.

We also consider Lévy-Itô operators

$$
\mathcal{I}_{L I}[u](x)=\int_{\mathbb{R}^{N}}\left(u(x+j(x, z))-u(x)-\nabla u(x) \cdot j(x, z) \mathbf{1}_{B}(z)\right) \mu(d z)
$$

where $\mu$ is a Lévy measure (hence it satisfies (4)) and $j(x, z)$ is the size of the jumps at $x$. Such operators enter in the general framework of (3) and the existence of $\mu_{x}$ is obtained through the representation of integral (5). In order that the operator is well-defined, one assumes

$$
|j(x, z)| \leq \bar{c}|z| \text { for some } \bar{c}>0 \text { and for any } x, z \in \mathbb{R}^{N} \text {. }
$$

In the definition of the Dirichlet problem, these terms have to be slightly modified and we refer the reader to Section 1 for a presentation of these (slight) modifications.

Roughly speaking, our aim is to find general conditions on $F$ and the non-local operator $\mathcal{I}$ ensuring that the (continuous) solutions of (1)-(2) (when they exist) satisfy $u=g$ on $\partial \Omega$ and then to show that such solutions do exist. In fact, in such problems, it is natural to introduce the function $\tilde{u}: \mathbb{R}^{N} \rightarrow \mathbb{R}$ defined by

$$
\tilde{u}(x)= \begin{cases}u(x) & \text { if } x \in \bar{\Omega} \\ g(x) & \text { otherwise }\end{cases}
$$

and another way of seeing our task is to say that we are going to investigate the continuity (or discontinuity) of $\tilde{u}$ on $\partial \Omega$. To simplify the notation, we keep writing $u$ instead of $\tilde{u}$ as far as the function defined in the whole space is concerned.

A first model equation is the linear equation

$$
-\operatorname{Tr}\left(a(x) D^{2} u\right)-b(x) \cdot D u-\mathcal{I}_{L}[u](x)+u=f(x) \quad \text { in } \Omega
$$

where $a, b, f$ are continuous functions defined on $\bar{\Omega}, a(x)$ being a symmetric non-negative matrix for any $x \in \bar{\Omega}$.

In the "local" case, i.e. when $\mu_{x} \equiv 0$, it is known that one can solve the Dirichlet problem in a classical way in smooth domains (either by probabilistic or viscosity solutions' methods) if $a$, $b$ and the signed distance function $d$ to $\partial \Omega$ (which is smooth in a neighborhood of $\partial \Omega, c f$. e.g. [12]) satisfy: for every $x \in \partial \Omega$,

(i) either $a(x) D d(x) \neq 0$ (strong ellipticity in the normal direction)

(ii) or $\operatorname{Tr}\left(a(x) D^{2} d(x)\right)+b(x) \cdot D d(x)<0$ (curvature and/or transport effects).

Among all the different proofs of this classical result, the viscosity solutions' proof ( $c f$. Barles \& Burdeau [3] or Da Lio [10] and references therein for more general results) shows that the effects of the ellipticity and of the drift (together with the curvature ones) are taken into account at 
different scales, the ellipticity ones having far stronger importance. One of the main interest of the present work is to analyze the effects of the non-local term. We will see that in the case of (1), we face two kinds of "elliptic"-type effects, acting also at different scales.

In order to give a general idea of the results we obtain in the linear case for instance, if one first considers the case of the pure fractional Laplace operator, that is if $d \mu_{x}(z)=d \mu(z)=d z /|z|^{N+\alpha}$ in (4) with $\alpha \in(0,2)$ and $a \equiv 0$ in (7), we then obtain the following result :

Let $a \equiv 0, b$ and $f$ be continuous. If either (i) $\alpha \geq 1$ or (ii) $b(x) \cdot \operatorname{Dd}(x)<0$ or (iii) $b \equiv 0$, then any solution of (7) takes the boundary data in the continous sense.

(In fact the results are more general even for the fractional Laplace operator, see Section 4). Moreover, if $0 \leq \alpha<1$, we show that if $b \not \equiv 0$ the boundary data may be lost (see Section 5); hence the corresponding result is somehow optimal.

If more general Lévy operators are at stake, quantities associated with the measures $\left(\mu_{x}\right)_{x \in \partial \Omega}$ play an important role. These quantities imbed the interplay between these singular measures and the geometry of the boundary. Roughly speaking, jumps along the normal must be under control.

Next, a general condition ensuring that the Dirichlet problem for a linear equation is satisfied in the classical sense can therefore be exhibited (see (21)), generalizing the result we gave above in the case of the fractional Laplace operator. It is worth pointing out that if there are "many" outer jumps, then the boundary condition will be satisfied in the classical sense. We also explain how to reduce the study of $C^{2}$-domains to domains with flat boundary (see Subsection 3.6).

Of course, the analysis of the non-local term allows us not only to provide results for the above simple linear equation (7) but also for a large class of non-linear equations under suitable growth conditions with respect to (wrt for short) $D u$.

We conclude this short (and very vague) presentation of the paper by mentioning that, if we are able to analyse the boundary behaviour of the sub- and supersolutions in a rather general framework, and in particular for rather general $x$-dependent measures in the non-local term, far more restrictive assumptions on $F$ and the non-local term are to be imposed in order to prove the existence of a (continuous) solution for the Dirichlet problem. Indeed, existence is obtained by applying Perron's method together with a comparison result for the integro-differential equation, and restrictive assumptions are to be made on the non-linearity and the singular measure in order to get such a comparison result.

We refer the reader to Ishii [14] or the User's guide [9] for the presentation of Perron's method which extends to the case of non-local equations because of the general stability result for integrodifferential equations of Bensaoud and Sayah [5] (see also [4]) and to [16, 15, 2, 4] and references therein for comparison results for second order elliptic integro-differential equations.

We point out that other existence results have been obtained by Arisawa [2] by assuming the existence of sub- and supersolutions agreeing with the boundary data on $\partial \Omega$ and we also want to mention the study of options with barriers by Cont and Voltchkova [8] which leads in dimension 1 to similar questions but addressed from a probabilistic point of view. Finally, for non-local equations associated with bounded measures and in an evolution setting, Chasseigne [7] shows that the Dirichlet problem can be solved completely although the Dirichlet boundary condition has to be considered in a generalized sense since it does not hold in the classical one.

Organization of the Paper. After defining viscosity solutions in Section 1, we provide existence and uniqueness results for Lévy-Itô operators. Section 2 is devoted to the key technical result of this paper. It is shown that, at points where the boundary condition is not satisfied in 
the classical sense, necessary conditions hold, involving first- and second-order differential terms and non-local ones. We next use this technical result to study the behaviour of solutions at the boundary in differents cases (Section 3). The results we give provide sufficient conditions in order that the Dirichlet condition is satisfied in the classical sense. We start with the simplest case, namely a linear equation involving the fractional Laplacian on the half-space and we generalize this result progressively to a large class of Lévy operators on smooth domains. We next treat further examples involving different non-linearities (Section 4). Eventually, we give in Section 5 an explicit example showing that if the measure is not singular enough near the origin, the behaviour of the solution at the boundary may change if a drift term appears in the equation; we will show that the boundary condition may not be satisfied in the classical sense anymore.

Acknowledments. The authors wish to thank Jean Bertoin for the fruitful discussions we had with him and the referees for their careful reading and precise comments. Both discussions and comments lead to a real improvement of the first version of this article. The discussions with Jean Bertoin gave us a better understanding of Lévy processes and, in particular, of the role of the compensator. They were a source of motivation: in this new version, the results are obtained under assumptions which are natural from a probabilistic point of view, which was not completely the case in the first one.

Notations. Throughout the paper, $B_{r}(x) \subset \mathbb{R}^{N}$ denotes the open ball of radius $r$ centered at $x$. If $x=0$, we simply write $B_{r}$. The open unit ball is denoted $B$ and $\mathbf{1}_{B}$ denotes the indicator function of $B$. When estimating non-local terms, we use also the following notation: $A \propto B$ means that $c_{1} B \leq A \leq c_{2} B$ for two constants $c_{1}, c_{2}>0$.

Recall that $\Omega$ is assumed to be a $C^{2}$-domain. This implies, in particular, that the signed distance function $d$ to $\partial \Omega$, which is non-negative in $\Omega$ and non-positive in its complementary, is $C^{2}$ in a neighbourhood of $\partial \Omega$. Furthermore, $d$ also denotes a $C^{2}$-function in $\mathbb{R}^{N}$ which agrees with $d$ in a neighbourhood of $\partial \Omega$.

\section{Viscosity solutions of the Dirichlet PRoblem}

We recall in this section the definition of viscosity sub- and supersolutions for the Dirichlet problem and we construct solutions by Perron's method. We point out that we use a formulation based on the most classical definition introduced by Sayah [18, 19] (see also [4] and reference therein). Notice that there exists an alternative approach [2] we will not use here.

1.1. Definition of viscosity solutions. We mentioned in the Introduction that sub- and supersolutions are seen as functions defined in $\mathbb{R}^{N}$ and equal to $g$ on $\Omega^{c}$ (or less/greater than $g$ on $\Omega^{c}$.

Definition 1. A usc function $u: \mathbb{R}^{N} \rightarrow \mathbb{R}$ is a subsolution of (1)-(2) if, for any test function $\phi \in C^{2}\left(\mathbb{R}^{N}\right)$, at each maximum point $x_{0} \in \bar{\Omega}$ of $u-\phi$ in $B_{\delta}\left(x_{0}\right)$, we have

$$
\mathrm{E}\left(u, \phi, x_{0}\right):=F\left(x_{0}, u\left(x_{0}\right), D \phi\left(x_{0}\right), D^{2} \phi\left(x_{0}\right), I_{\delta}^{1}[\phi]\left(x_{0}\right)+I_{\delta}^{2}[u]\left(x_{0}\right)\right) \leq 0 \quad \text { if } x_{0} \in \Omega,
$$

or

$$
\min \left(\mathrm{E}\left(u, \phi, x_{0}\right) ; u\left(x_{0}\right)-g\left(x_{0}\right)\right) \leq 0 \quad \text { if } x_{0} \in \partial \Omega
$$


where

$$
\begin{aligned}
& I_{\delta}^{1}[\phi]\left(x_{0}\right)=\int_{\{|z|<\delta\}}\left\{\phi\left(x_{0}+z\right)-\phi\left(x_{0}\right)-\left(D \phi\left(x_{0}\right) \cdot z\right) \mathbf{1}_{B}(z)\right\} d \mu_{x_{0}}(z) \\
& I_{\delta}^{2}[u]\left(x_{0}\right)=\int_{\{|z| \geq \delta\}}\left\{u\left(x_{0}+z\right)-u\left(x_{0}\right)-\left(D \phi\left(x_{0}\right) \cdot z\right) \mathbf{1}_{B}(z)\right\} d \mu_{x_{0}}(z) .
\end{aligned}
$$

A lsc function $v: \mathbb{R}^{N} \rightarrow \mathbb{R}$ is a supersolution of (1)-(2) if, for any test function $\phi \in C^{2}\left(\mathbb{R}^{N}\right)$, at each minimum point $x_{0} \in \bar{\Omega}$ of $v-\phi$ in $B_{\delta}\left(x_{0}\right)$, we have

$$
\mathrm{E}\left(v, \phi, x_{0}\right):=F\left(x_{0}, v\left(x_{0}\right), D \phi\left(x_{0}\right), D^{2} \phi\left(x_{0}\right), I_{\delta}^{1}[\phi]\left(x_{0}\right)+I_{\delta}^{2}[v]\left(x_{0}\right)\right) \geq 0 \quad \text { if } x_{0} \in \Omega,
$$

or

$$
\max \left(\mathrm{E}\left(v, \phi, x_{0}\right) ; v\left(x_{0}\right)-g\left(x_{0}\right)\right) \geq 0 \quad \text { if } x_{0} \in \partial \Omega .
$$

Finally, a viscosity solution of (1)-(2) is a function whose upper and lower semicontinuous envelopes are respectively sub- and supersolution of the problem.

1.2. Existence and uniqueness of viscosity solutions. We now turn to the existence issue for (1)-(2). We provide such a result in the case of Lévy-Itô measures and we do it by assuming $F$ to be defined in $\mathbb{R}^{N} \times \mathbb{R} \times \mathbb{R}^{N} \times \mathbb{S}^{N} \times \mathbb{R}$ (instead of $\bar{\Omega} \times \mathbb{R} \times \mathbb{R}^{N} \times \mathbb{S}^{N} \times \mathbb{R}$ ) to simplify the exposition.

The assumptions we use are the following ones (see the remark below for some comments).

(A1) The measure $\mu(d z)$ and the function $j(x, z)$ satisfy: there exists a constant $\bar{c}>0$ such that

$$
\begin{gathered}
\int_{B}|j(x, z)|^{2} \mu(d z)<+\infty, \int_{\mathbb{R}^{N} \backslash B} \mu(d z)<+\infty \\
\int_{\mathbb{R}^{N}}|j(x, z)-j(y, z)|^{2} \mu(d z) \leq \bar{c}|x-y|^{2} \text { and } \int_{\mathbb{R}^{N} \backslash B}|j(x, z)-j(y, z)| \mu(d z) \leq \bar{c}|x-y| .
\end{gathered}
$$

(A2) There exists $\gamma>0$ such that for any $x \in \mathbb{R}^{N}, u, v \in \mathbb{R}, p \in \mathbb{R}^{N}, X \in \mathbb{S}^{N}$ and $l \in \mathbb{R}$

$$
F(x, u, p, X, l)-F(x, v, p, X, l) \geq \gamma(u-v) \quad \text { when } \quad u \geq v .
$$

(A3-1) For any $R>0$, there exist moduli of continuity $\omega$ (independent of $R$ ) and $\omega_{R}$ such that, for any $|x|,|y| \leq R,|v| \leq R, l \in \mathbb{R}$ and for any $X, Y \in \mathbb{S}^{N}$ satisfying

$$
\left[\begin{array}{cc}
X & 0 \\
0 & -Y
\end{array}\right] \leq \frac{1}{\varepsilon}\left[\begin{array}{cc}
I & -I \\
-I & I
\end{array}\right]+r(\beta)\left[\begin{array}{ll}
I & 0 \\
0 & I
\end{array}\right]
$$

for some $\varepsilon>0$ and $r(\beta) \rightarrow 0$ as $\beta \rightarrow 0$, then, if $s(\beta) \rightarrow 0$ as $\beta \rightarrow 0$, we have

$$
F\left(y, v, \varepsilon^{-1}(x-y), Y, l\right)-F\left(x, v, \varepsilon^{-1}(x-y)+s(\beta), X, l\right) \leq \omega(\beta)+\omega_{R}\left(|x-y|+\varepsilon^{-1}|x-y|^{2}\right)
$$

or

(A3-2) For any $R>0, F$ is uniformly continuous on $\mathbb{R}^{N} \times[-R, R] \times B_{R} \times D_{R} \times \mathbb{R}$ where $D_{R}:=\left\{X \in \mathbb{S}^{N} ;|X| \leq R\right\}$ and there exist a modulus of continuity $\omega_{R}$ such that, for any $x, y \in \mathbb{R}^{N},|v| \leq R, l \in \mathbb{R}$ and for any $X, Y \in \mathbb{S}^{N}$ satisfying (8) and $\varepsilon>0$, we have

$$
F\left(y, v, \varepsilon^{-1}(x-y), Y, l\right)-F\left(x, v, \varepsilon^{-1}(x-y), X, l\right) \leq \omega_{R}\left(\varepsilon^{-1}|x-y|^{2}+|x-y|+r(\beta)\right) .
$$

(A4) $F(x, u, p, X, l)$ is Lipschitz continuous in $l$, uniformly with respect to all other variables.

(A5) $\sup _{x \in \mathbb{R}^{N}}|F(x, 0,0,0,0)|<+\infty$.

(A6) For any $x \in \partial \Omega$, the infimum limit in (10) is strictly positive and the supremum limit in (11) is strictly negative. 
We can now state the existence and uniqueness result.

Theorem 1. Assume that (A1), (A2), (A4), (A5) and (A6) hold. Then there exists a unique bounded, continuous solution of the Dirichlet problem (1)-(2) if, in addition, one of the following set of conditions is fullfilled

(i) (A3-1) holds and $g$ is a bounded continuous function in $\mathbb{R}^{N}$,

(ii) (A3-2) holds, $\Omega$ is a bounded open subset of $\mathbb{R}^{N}$ and $g$ is a bounded uniformly continuous function in $\mathbb{R}^{N}$.

Moreover, in both cases, one has a comparison result for this problem in the class of bounded sub- and supersolutions.

The main interest of this result is to provide the existence of a continuous (up to the boundary) viscosity solution of the Dirichlet problem without assuming the existence of sub- and supersolutions (ordered in the right way). Such an assumption is very often made when one wants to use the Perron's method, see for instance Arisawa [2]. Notice that it may be rather difficult to build such sub- and supersolutions for integro-differential problems; it is done when proving Theorem 1 thanks to the key technical lemma of Section 2.

Remark 1. Let us make a few comments about the assumptions. Of course, (A2) is very classical and, unfortunately, the combination of (A3)-(A4) does not allow a very general form and dependence of the non-local term: in particular, we do not know how to handle operators of the general form (3).

As far as (A3) is concerned, (A3-1) allows more general dependence wrt $x$ than (A3-2), which, on the contrary, allows more general dependence wrt $p$, and this is reflected in Theorem 1 by the cases (i) and (ii) for reasons which will be clear in the proof (at least, we hope so!).

We refer to [4] for more comments on these assumptions ensuring that a comparison result holds true in the whole space $\mathbb{R}^{N}$.

Proof of Theorem 1. We consider two bounded continuous functions $\psi_{1}, \psi_{2}: \mathbb{R}^{N} \rightarrow \mathbb{R}$ such that $\psi_{1} \geq \psi_{2}$ in $\mathbb{R}^{N}$ and $\psi_{1}=\psi_{2}=g$ on $\Omega^{c}$. Notice that they are uniformly continuous in the case of (ii).

We set

$$
\tilde{F}(x, u, p, X, l):=\min \left(u-\psi_{2} ; \max \left(u-\psi_{1} ; F(x, u, p, X, l)\right) .\right.
$$

It is straightforward to show that $\tilde{F}$ still satisfies (A1), (A2), (A3), (A4), (A5) and this is where the difference between (i) and (ii) plays a role: indeed, in the case of (i), $\psi_{1}, \psi_{2}$ are just bounded continuous functions and this is consistent with (A3-1), while, in the case of (ii), they are bounded uniformly continuous functions which is consistent with (A3-2).

If $M=\max \left(\|g\|_{\infty} ; \gamma^{-1}\|F(x, 0,0,0,0)\|_{\infty}\right)$, then $-M$ and $+M$ are respectively sub- and supersolution of the equation $\tilde{F}=0$ in $\mathbb{R}^{N}$. By applying the Perron's Method $(c f$. [9, 14] for local equations and $[4,19,1,5,13]$ for non-local ones) together with the comparison result of [4] provides the existence of a solution $u$ for this integro-differential equation such that $|u| \leq M$.

In order to prove the existence of a solution for the Dirichlet problem, the idea is to choose an increasing sequence of functions $\left(\psi_{1}^{\alpha}\right)_{\alpha}$ and a decreasing sequence of functions $\left(\psi_{2}^{\alpha}\right)_{\alpha}$ such that $\psi_{1}^{\alpha}(x) \rightarrow+\infty$ and $\psi_{2}^{\alpha}(x) \rightarrow-\infty$ for any $x \in \Omega$. Since the associated solutions $\left(u^{\alpha}\right)_{\alpha}$ we constructed above are uniformly bounded by $M$, the half-relaxed limit method provides us with an usc subsolution $\bar{u}$ and a lsc supersolution $\underline{u}$ of the Dirichlet problem (in the sense of Definition 1) such that $\bar{u} \geq \underline{u}$ in $\mathbb{R}^{N}$. 


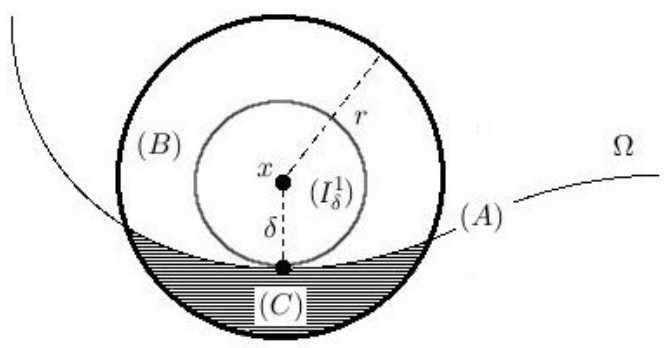

FiguRE 1. Domain decomposition

But, by (A6) and Lemma 1, one has $g \leq \underline{u} \leq \bar{u} \leq g$ on $\Omega^{c}$ and therefore $\bar{u}, \underline{u}$ are continuous on $\partial \Omega$ with $\underline{u}=\bar{u}=g$ on $\partial \Omega$. Using this property, it is easy to build $\psi_{1}, \psi_{2}$ such that $\psi_{2} \leq \underline{u}, \bar{u} \leq \psi_{1}$ on $\bar{\Omega}$ and $\psi_{1}=\psi_{2}=g$ on $\Omega^{c}$; moreover, $\psi_{1}, \psi_{2}$ can be built in order to be bounded continuous functions in the case of (i) and bounded uniformly continuous functions in the case of (ii).

In both cases, $\bar{u}, \underline{u}$ are respectively viscosity sub- and supersolution of the equation of the type $\tilde{F}=0$ in $\mathbb{R}^{N}$ associated with $\psi_{1}, \psi_{2}$ for which we have a comparison result by the same arguments as above. Therefore $\bar{u} \leq \underline{u}$ in $\mathbb{R}^{N}$. Finally $u:=\bar{u}=\underline{u}$ is the solution of the Dirichlet problem we were looking for and the Dirichlet condition is satisfied in the classical sense.

Remark 2. A similar argument provides the comparison result for the Dirichlet problem.

\section{A Key technicAl LEMma}

This section is dedicated to the statement and proof of the main technical result of this article: it concerns the conditions on $F$ and the integral operator which have to be satisfied either to allow a loss of boundary condition or, on the contrary, under which they cannot occur.

In order to present this result, we need to decompose the integral operator into several parts, each one corresponding to a region of $\mathbb{R}^{N}$ and an integrand. More precisely, for $0<\delta, \beta<r$ and $x \in \bar{\Omega}$ close to the boundary $\partial \Omega$, we write (see Figure 1 ):

$$
\mathbb{R}^{N}=B_{r}^{c} \cup \mathcal{A}_{\beta, r}^{\mathrm{int}}(x) \cup \mathcal{A}_{\delta, r}^{\mathrm{ext}}(x) \cup \mathcal{A}_{\delta, \beta, r}(x)
$$

where

$$
\begin{gathered}
\mathcal{A}_{\delta, \beta, r}(x)=\left\{z \in B_{r}:-\delta \leq d(x+z)-d(x) \leq \beta\right\}, \\
\mathcal{A}_{\delta, r}^{\mathrm{ext}}(x)=\left\{z \in B_{r}: d(x+z)-d(x)<-\delta\right\}, \\
\mathcal{A}_{\beta, r}^{\mathrm{int}}(x)=\left\{z \in B_{r}: d(x+z)-d(x)>\beta\right\} .
\end{gathered}
$$

The notation "ext/int" stands for "exterior/interior" since, in fact, these sets will correspond below to points $x+z$ which are either outside or inside the domain $\Omega$; this is clear for $\mathcal{A}_{\beta, r}^{\text {int }}(x)$ because $d(x+z)>d(x)+\beta>0$ and, for $\mathcal{A}_{\delta, r}^{\text {ext }}(x)$, this will be a consequence of the choice $\delta \geq d(x)$. We also point out that $B_{\delta} \subset \mathcal{A}_{\delta, \beta, r}(x)$ and $\mathcal{A}_{\beta, r}^{\mathrm{int}}(x) \subset\left(B_{\beta}\right)^{c}$. 
Next we can define integral quantities associated with different integrands and different subsets.

$$
\left\{\begin{aligned}
I_{\delta, r}^{\mathrm{ext}, 1}(x) & =\int_{\mathcal{A}_{\delta, r}^{\mathrm{ext}}(x)} d \mu_{x}(z), \\
I_{\delta, r}^{\mathrm{ext}, 2}(x) & =\int_{\mathcal{A}_{\delta, r}^{\mathrm{ext}}(x)} D d(x) \cdot z d \mu_{x}(z), \\
I_{\delta, r}^{\mathrm{ext}, 3}(x) & =\int_{\mathcal{A}_{\delta, r}^{\text {ext }}(x)}|z| d \mu_{x}(z), \\
I_{\beta, r}^{\mathrm{int}, 1}(x) & =\int_{\mathcal{A}_{\beta, r}^{\mathrm{int}}(x)} d \mu_{x}(z), \\
I_{\beta, r}^{\mathrm{int}, 2}(x) & =\int_{\mathcal{A}_{\beta, r}^{\mathrm{int}}(x)}^{\mathrm{int}} D d(x) \cdot z d \mu_{x}(z), \\
I_{\beta, r}^{\mathrm{int}, 3}(x) & =\int_{\mathcal{A}_{\beta, r}^{\mathrm{int}}(x)}|z| d \mu_{x}(z), \\
I_{\delta, \beta, r}^{4}(x) & =\frac{1}{2} \int_{\mathcal{A}_{\delta, \beta, r}(x)}|z|^{2} d \mu_{x}(z), \\
I^{\mathrm{tr}}(x) & =\int_{r<|z|<1} D d(x) \cdot z d \mu_{x}(z)
\end{aligned}\right.
$$

We can now state the technical lemma.

Lemma 1. Let $u$ be a bounded usc viscosity subsolution of the Dirichlet problem (1)-(2) and $R:=\|u\|_{\infty}$. If, for some $x \in \partial \Omega$, we have $u(x)>g(x)$, there then exist $\nu, r_{0}>0$ such that, for any $k_{1}, k_{2}>0$, any $r \in\left(0, r_{0}\right)$ and any sequences $\beta(\eta), \varepsilon(\eta) \rightarrow 0$ as $\eta \rightarrow 0$, we have

$$
\liminf _{\substack{y \rightarrow x, y \in \bar{\Omega} \\ \eta \downarrow 0, d(y) \eta^{-1} \rightarrow 0}}\left\{\sup _{\substack{\delta \in[d(y), r) \\ \delta>0}} \inf _{\substack{y \in[-R, R] \\ \text { (n) }}}\left[F\left(y, s, p_{\eta}(y), M_{\eta}(y), I_{\eta, \delta, r}(y)\right)\right]\right\} \leq 0,
$$

where

$$
\begin{aligned}
p_{\eta}(y)= & O\left(\frac{1}{\varepsilon}\right)+\frac{k_{1}+o(1)}{\eta} D d(y), \\
M_{\eta}(y)= & O\left(\frac{1}{\varepsilon}\right)+\frac{k_{1}+o(1)}{\eta} D^{2} d(y)-\frac{k_{2}+o(1)}{\eta^{2}} D d(y) \otimes D d(y), \\
I_{\eta, \delta, r}(y)= & -\nu I_{\delta, r}^{\mathrm{ext}, 1}(y)+2\|u\|_{\infty} I_{\beta(\eta), r}^{\mathrm{int}, 1}(y) \\
& -\frac{k_{1}+o(1)}{\eta}\left(I^{\mathrm{tr}}(y)+I_{\beta(\eta), r}^{\mathrm{int}, 2}(y)+I_{\delta, r}^{\mathrm{ext}, 2}(y)-\left\|D^{2} d\right\|_{\infty} I_{\delta, \beta(\eta), r}^{4}(y)\right) \\
& +O\left(\frac{1}{\varepsilon}\right)\left(1+o(1) I_{\beta(\eta), r}^{\mathrm{int}, 3}(y)+o(1) I_{\delta, r}^{\mathrm{ext}, 3}(y)\right)
\end{aligned}
$$

where $O\left(\varepsilon^{-1}\right)$ does not depend on $k_{1}$ and $k_{2}$.

Let $v$ be a bounded lsc viscosity supersolution of the Dirichlet problem and $R:=\|v\|_{\infty}$. If, for some $x \in \partial \Omega$, we have $v(x)<g(x)$, there then exist $\nu, r_{0}>0$ such that, for any $k_{1}, k_{2}>0$, any $r \in\left(0, r_{0}\right)$ and any sequences $\beta(\eta), \varepsilon(\eta) \rightarrow 0$ as $\eta \rightarrow 0$, we have

$$
\limsup _{\substack{y \rightarrow x, y \in \bar{\Omega} \\ \eta \downarrow 0, d(y) \eta^{-1} \rightarrow 0}}\left\{\inf _{\substack{\delta \in[(y), r) \\ \delta>0}} \sup _{s \in[-R, R]}\left[F\left(y, s,-p_{\eta}(y),-M_{\eta}(y),-I_{\eta, \delta, r}(y)\right)\right]\right\} \geq 0 .
$$

Remark 3. We have considered in Lemma 1 non-local terms written as Lévy operators but in stochastic control with jump processes (see for instance [17]), one can also consider the so-called 
Lévy-Itô diffusions whose infinitesimal generators are of the form (5). It is worth pointing out that Lemma 1 can be readily translated for such operators by using appropriate $x$-dependent measures $\mu_{x}(d z)$ associated with $\mu$ and $j(x, \cdot)$. See below for further details.

Proof of Lemma 1. We only provide the proof for the subsolution case, since the supersolution one is analogous.

We set $4 \nu:=u(x)-g(x)>0$. Since $g$ is continuous, there exists $r_{0}=r_{0}(x)>0$ such that, for all $y \in B\left(x, 2 r_{0}\right) \cap \Omega^{c}$,

$$
|g(x)-g(y)| \leq \nu .
$$

Next we introduce the test-function

$$
\psi(y):=\frac{\chi(y-x)}{\varepsilon}+\varphi\left(\frac{d(y)}{\eta}\right)
$$

where $\chi: \mathbb{R}^{N} \rightarrow \mathbb{R}$ is a smooth bounded function such that $\chi(0)=0$ and $\chi(y)>0$ if $y \neq 0$, $\liminf _{|y| \rightarrow \infty} \chi(y)>0$, and $D^{2} \chi$ is bounded, while $\varphi: \mathbb{R} \rightarrow \mathbb{R}$ is a bounded, smooth, increasing function, which is concave on $(0,+\infty)$ and such that $\varphi(0)=0, \varphi^{\prime}(0)=k_{1}, \varphi^{\prime \prime}(0)=-k_{2}$ with $k_{1}, k_{2}>0$ and $\varphi \geq-\nu$ in $\mathbb{R}$. For the sake of clarity, we have dropped the dependence of $\psi$ in $\varepsilon$ and $\eta$ and we will do so too for the maximum points we consider below. We can think of these conditions as if $\varepsilon$ depends on $\eta$ and $\varepsilon(\eta) \rightarrow 0$ as $\eta \rightarrow 0$, even if we also drop this dependence for the sake of simplicity of notations.

Keeping in mind the way we have chosen $\chi$ and $\varphi$, we know that for $\eta$ (hence $\varepsilon$ ) small enough, $y \mapsto u(y)-\psi(y)$ achieves its global maximum at a point $\bar{x}$. Since $\varphi$ is bounded, we deduce from $(u-\psi)(x) \leq(u-\psi)(\bar{x})$ that the following inequality holds true

$$
\frac{\chi(x-\bar{x})}{\varepsilon} \leq u(\bar{x})-u(x)+2\|\varphi\|_{\infty} .
$$

In particular, $\frac{\chi(\bar{x}-x)}{\varepsilon}$ remains bounded as $\eta \rightarrow 0$ and therefore $\bar{x} \rightarrow x$. In particular, $\bar{x} \in$ $B\left(x, 2 r_{0}\right)$ for $\eta$ small enough. We also get

$$
g(x)+4 \nu=u(x) \leq u(\bar{x})-\frac{\chi(\bar{x}-x)}{\varepsilon}-\varphi\left(\frac{d(\bar{x})}{\eta}\right) \leq u(\bar{x})+2 \nu
$$

(remember that $\chi \geq 0$ and $\varphi \geq-\nu$ in $\mathbb{R}$ ). Therefore, for $\eta$ small enough, $\bar{x}$ is necessarily in $\bar{\Omega}$, otherwise the above inequality would contradict (12).

This implies $d(\bar{x}) \geq 0$ and therefore, since the $\chi$-term is non-negative, we have $\varphi(d(\bar{x}) / \eta) \leq$ $u(\bar{x})-u(x)$ from which we deduce, using the upper semicontinuity of $u$, that, as $\eta \rightarrow 0$,

$$
\varphi\left(\frac{d(\bar{x})}{\eta}\right) \rightarrow 0 \quad \text { i.e. } \quad \frac{d(\bar{x})}{\eta} \rightarrow 0 \quad \text { and } \quad u(\bar{x}) \rightarrow u(x) .
$$

We may have either $\bar{x} \in \Omega$ or $\bar{x} \in \partial \Omega$. In the first case, the $F$-viscosity inequality obviously holds but we claim that it holds also in the second case if $\eta$ is small enough: indeed, if $\bar{x} \in \partial \Omega$, since $u(\bar{x}) \rightarrow u(x)=g(x)+4 \nu$ as $\eta \rightarrow 0$, we clearly have $u(\bar{x})>g(\bar{x})$ for $\eta$ small enough by the continuity of $g$.

Therefore, by Definition 1 , we can write the following $F$-viscosity inequality in both cases

$$
F\left(\bar{x}, u(\bar{x}), D \psi(\bar{x}), D^{2} \psi(\bar{x}), I_{\delta}^{1}[\psi](\bar{x})+I_{\delta}^{2}[u](\bar{x})\right) \leq 0
$$


for some $\delta>0$ small enough to be chosen later and where

$$
\begin{aligned}
I_{\delta}^{1}[\psi](\bar{x}) & =\int_{\{|z|<\delta\}}\left\{\psi(\bar{x}+z)-\psi(\bar{x})-(D \psi(\bar{x}) \cdot z) \mathbf{1}_{B}(z)\right\} d \mu_{\bar{x}}(z), \\
I_{\delta}^{2}[u](\bar{x}) & =\int_{\{|z| \geq \delta\}}\left\{u(\bar{x}+z)-u(\bar{x})-(D \psi(\bar{x}) \cdot z) \mathbf{1}_{B}(z)\right\} d \mu_{\bar{x}}(z) .
\end{aligned}
$$

The proof consists now in estimating all the terms of this equation, and in particular non-local ones.

As far as derivatives are concerned, since $D \chi(0)=0$ and thanks to the properties of $\bar{x}$ and $\varphi$, we have

$$
\begin{aligned}
D \psi(\bar{x}) & =\frac{o(1)}{\varepsilon}+\frac{k_{1}+o(1)}{\eta} \operatorname{Dd}(\bar{x}), \\
D^{2} \psi(\bar{x}) & =\frac{O(1)}{\varepsilon}+\frac{k_{1}+o(1)}{\eta} D^{2} d(\bar{x})-\frac{k_{2}+o(1)}{\eta^{2}} \operatorname{Dd}(\bar{x}) \otimes D d(\bar{x}) .
\end{aligned}
$$

We now turn to non-local terms. Using the fact that $\bar{x}$ is a global maximum point of $u-\psi$, we next have, for any $z \in \mathbb{R}^{N}$

$$
u(\bar{x}+z)-u(\bar{x}) \leq \psi(\bar{x}+z)-\psi(\bar{x}) ;
$$

the non-local term $I_{\delta}^{1}[\psi](\bar{x})+I_{\delta}^{2}[u](\bar{x})$ can thus be estimated from above as the sum of four terms

$$
I_{\delta}^{1}[\psi](\bar{x})+I_{\delta}^{2}[u](\bar{x}) \leq(A)+(B)+(C)+(D)
$$

where

$$
\begin{aligned}
(A) & =\int_{\{|z| \geq r\}}\left\{u(\bar{x}+z)-u(\bar{x})-(D \psi(\bar{x}) \cdot z) \mathbf{1}_{B}(z)\right\} d \mu_{\bar{x}}(z) \\
(B) & =\int_{\mathcal{A}_{\beta(\eta), r}^{\text {int }}(\bar{x})}\left\{u(\bar{x}+z)-u(\bar{x})-(D \psi(\bar{x}) \cdot z) \mathbf{1}_{B}(z)\right\} d \mu_{\bar{x}}(z) \\
(C) & =\int_{\mathcal{A}_{\delta, r}^{\text {ext }}(\bar{x})}\left\{g(\bar{x}+z)-u(\bar{x})-(D \psi(\bar{x}) \cdot z) \mathbf{1}_{B}(z)\right\} d \mu_{\bar{x}}(z) \\
(D) & =\int_{\mathcal{A}_{\delta, \beta(\eta)}(\bar{x})}\left\{\psi(\bar{x}+z)-\psi(\bar{x})-(D \psi(\bar{x}) \cdot z) \mathbf{1}_{B}(z)\right\} d \mu_{\bar{x}}(z),
\end{aligned}
$$

(recall that $B_{\delta} \subset \mathcal{A}_{\delta, \beta(n)}(\bar{x})$ ). In these terms, we make the following choices: $\delta>0$ satisfies also $\delta \geq d(\bar{x})$ (therefore the "ext" term actually takes into account points $x+z$ which are in $\Omega^{c}$ ) and we choose $0<r<r_{0}$, independent of $\eta$ (we recall that $r_{0}$ is given by (12)). The parameter $\beta(\eta)>0$ is at least chosen such that $\beta(\eta)<r$.

Now we estimate successively each term.

Estimate of $(A)$ : Since $u$ is bounded and $\sup _{\bar{x}} \int_{\mathbb{R}^{N} \backslash B(0, r)} d \mu_{\bar{x}}(z)<\infty$, we obtain by using (13)

$$
(A) \leq O\left(\varepsilon^{-1}\right)-\frac{k_{1}+o(1)}{\eta} I^{\operatorname{tr}}(\bar{x})
$$

Here we have used the fact that $r$ is fixed.

Estimate of $(B)$ : Estimating each term in a straightforward way, we have

$$
(B) \leq 2\|u\|_{\infty} I_{\beta(\eta), r}^{\mathrm{int}, 1}(\bar{x})-\frac{k_{1}+o(1)}{\eta} I_{\beta(\eta), r}^{\mathrm{int}, 2}(\bar{x})+o\left(\varepsilon^{-1}\right) I_{\beta(\eta), r}^{\mathrm{int}, 3}(\bar{x}) .
$$


Estimate of $(C)$ : In this term, we take into account the jump of $u$ at the boundary. Recalling the fact that $u(\bar{x}) \rightarrow u(x)$, we can choose $\eta$ small enough so that $|u(\bar{x})-u(x)| \leq \nu$. Using (12) and the definition of $r$, this implies, in particular, that

$$
\text { for all } z \in B_{r} \text { such that } \bar{x}+z \in \Omega^{c}, \quad g(\bar{x}+z)-u(\bar{x}) \leq-\nu
$$

and we then conclude that we have

$$
(C) \leq-\nu I_{\delta, r}^{\mathrm{ext}, 1}(\bar{x})-\frac{k_{1}+o(1)}{\eta} I_{\delta, r}^{\mathrm{ext}, 2}(\bar{x})+o\left(\varepsilon^{-1}\right) I_{\delta, r}^{\mathrm{ext}, 3}(\bar{x}) .
$$

Estimate OF (D): recalling that $\varphi$ is concave on $(0,+\infty)$, we have

$$
\begin{aligned}
(D) & \leq \frac{1}{\eta} \varphi^{\prime}\left(\frac{d(\bar{x})}{\eta}\right) \int_{\mathcal{A}_{\delta, \beta(\eta), r}(\bar{x})}\left(d(\bar{x}+z)-d(\bar{x})-D d(\bar{x}) \cdot z \mathbf{1}_{B}(z)\right) \mu_{\bar{x}}(d z)+O\left(\varepsilon^{-1}\right) \\
& \leq \frac{k_{1}+o(1)}{\eta}\left\|D^{2} d\right\|_{\infty} I_{\delta, \beta(\eta), r}^{4}(\bar{x})+O\left(\varepsilon^{-1}\right) .
\end{aligned}
$$

Here we have used the fact that the signed distance function $d$ is $C^{2}$ to get the last line.

Gathering all these estimates in the viscosity subsolution's inequality and using the fact that $F$ is non-increasing with respect to its last argument, we finally have

$$
F\left(\bar{x}, u(\bar{x}), p_{\eta}(\bar{x}), M_{\eta}(\bar{x}), I_{\delta, \beta(\eta), r}(\bar{x})\right) \leq 0
$$

where

$$
\begin{aligned}
I_{\delta, \beta(\eta), r}(\bar{x})= & -\nu I_{\delta, r}^{\mathrm{ext}, 1}+2\|u\|_{\infty} I_{\beta(\eta), r}^{\mathrm{int}, 1}(\bar{x}) \\
& -\frac{k_{1}+o(1)}{\eta}\left(I^{\mathrm{tr}}(\bar{x})+I_{\beta(\eta), r}^{\mathrm{int}, 2}(\bar{x})+I_{\delta, r}^{\mathrm{ext}, 2}-\left\|D^{2} d\right\|_{\infty} I_{\delta, \beta(\eta), r}^{4}(\bar{x})\right) \\
& +O\left(\frac{1}{\varepsilon}\right)\left(1+o(1) I_{\beta(\eta), r}^{\mathrm{int}, 3}(\bar{x})+o(1) I_{\delta, r}^{\mathrm{ext}, 3}(\bar{x})\right) .
\end{aligned}
$$

This achieves the proof of the lemma.

\section{THE LiNeAR MOdEL EQUATiON}

In this section, we first study the boundary behaviour of a viscosity solution in a model framework by considering (7)-(2) where $\mathcal{I}[u]$ is an operator of Lévy type, i.e. given by (3). We first mainly treat the case of the fractional Laplace operator, for which we recall that the Lévy measure is given by

$$
d \mu_{x}(z)=\frac{d z}{|z|^{N+\alpha}}
$$

where $0<\alpha<2$. We also first consider a flat domain $\Omega$. We next generalize the results to more general operators and general smooth domains.

3.1. Dirichlet problem on a half-space. Let us first begin by considering the model problem (7) in the half-space $\Omega=\left\{x_{N}>0\right\}$. We recall that we assume in this case that $a, b, f$ are continuous functions defined on $\bar{\Omega}, a(x)$ being a symmetric non-negative matrix for any $x \in \bar{\Omega}$. Let us mention that here $d(x)=x_{N}$, but we keep the notation $d(x)$ in order to generalize the result easily (see the following sections).

The question is whether a viscosity sub- or supersolution $u$ of (7)-(2) satisfies the Dirichlet boundary condition in the classical sense or not. 
The answer to this question we provide below, as the other similar results we obtain next, relies on the technical lemma we stated in the previous section (Lemma 1). It provides us with a necessary condition in the case where $u \neq g$ at the boundary involving both differential terms (corresponding to $D u$ and $D^{2} u$ ) and integral terms.

We only consider the subsolution case since the supersolution one can be treated analogously and leads to the same conditions since the equation is linear. We want to prove that $u \leq g$ on $\partial \Omega$ and we argue by contradiction assuming that it is not true. Hence (10) provides us for $r$ small enough some $y \in B_{r}(x)$ such that $d(y)=o(\eta)$ and for any $\delta \in[d(y), r), \delta>0$,

$$
\begin{aligned}
& \frac{k_{2}+o(1)}{\eta^{2}} a(y) D d(y) \cdot D d(y)-\frac{k_{1}+o(1)}{\eta}\left(b(y) \cdot D d(y)+\operatorname{Tr}\left(a(y) D^{2} d(y)\right)-I^{\operatorname{tr}}(y)\right) \\
& +\left[\nu I_{\delta, r}^{\mathrm{ext}, 1}(y)-\frac{k_{1}+o(1)}{\eta} I_{\delta, r}^{\mathrm{ext}, 2}-\frac{o(1)}{\varepsilon} I_{\delta, r}^{\mathrm{ext}, 3}(y)\right]+ \\
& {\left[-2\|u\|_{\infty} I_{\beta(\eta), r}^{\mathrm{int}, 1}(y)+\frac{k_{1}+o(1)}{\eta} I_{\beta(\eta), r}^{\mathrm{int}, 2}(y)-\frac{o(1)}{\varepsilon} I_{\beta(\eta), r}^{\mathrm{int}, 3}(y)\right] \leq \frac{k_{1}+o(1)}{\eta}\left\|D^{2} d\right\|_{\infty} I_{\delta, \beta(\eta), r}^{4}(y)+O\left(\frac{1}{\varepsilon}\right)}
\end{aligned}
$$

where we recall that the $O\left(\frac{1}{\varepsilon}\right)$ does not depend on $k_{1}$ and $k_{2}$.

Our aim is to reach a contradiction out of this inequality; to do so, we have written it in order to gather in the left-hand side the terms which play the main role, while the other ones are in the right-hand side. To justify this claim and to look for the "natural" assumptions on the non-local terms, we are going to consider the case of the fractional Laplacian.

3.2. The case of the fractional Laplacian. In the following lemma, we provide estimates on each integral term in the case where $\Omega$ is a half-space and the integral operator is the fractional Laplacian.

Lemma 2. For any $0<\alpha<2$ and for fixed $r>0$, as $\delta, \beta \rightarrow 0$ (with $0<\delta<\beta$ ), we have

$$
\begin{aligned}
I_{\delta, r}^{\mathrm{ext}, 1}(x) & =I_{\delta, r}^{\mathrm{int}, 1}(x)=\int_{\substack{z_{N}<-\delta \\
|z|<r}} \frac{d z}{|z|^{N+\alpha}} \propto \delta^{-\alpha} \\
I_{\delta, r}^{\mathrm{int}, 2}(x) & =-I_{\delta, r}^{\mathrm{ext}, 2}(x)=\int_{\substack{z_{N}>\delta \\
|z|<r}}\left|z_{N}\right| \frac{d z}{|z|^{N+\alpha}} \propto \begin{cases}\delta^{1-\alpha} & \text { if } \alpha>1, \\
\ln \frac{1}{\delta} & \text { if } \alpha=1, \\
1 & \text { if } \alpha<1,\end{cases} \\
I_{\delta, r}^{\mathrm{ext}, 3}(x) & =I_{\beta, r}^{\mathrm{int}, 3}(x)=\int_{\substack{z_{N}<-\delta \\
|z|<r}} \frac{d z}{|z|^{N+\alpha-1}} \propto \begin{cases}\delta^{1-\alpha} & \text { if } \alpha>1, \\
\ln \frac{1}{\delta} & \text { if } \alpha=1, \\
1 & \text { if } \alpha<1,\end{cases} \\
I_{\delta, \beta, r}^{4}(x) & =\frac{1}{2} \int_{\substack{-|z|<r \\
-z_{N}<\beta}} \frac{\left.d z\right|^{N+\alpha-2}}{|z| z} \begin{array}{ll}
\beta^{2-\alpha} & \text { if } \alpha>1, \\
\beta \ln \frac{1}{\beta} & \text { if } \alpha=1, \\
\beta & \text { if } \alpha<1,
\end{array} \\
I^{\operatorname{tr}}(x) & =\int_{r<|z|<1} z_{N} \frac{d z}{|z|^{N+\alpha}}=0 .
\end{aligned}
$$

Proof. We assume for clarity (and without loss of generality) that $r=1$. Let us first compute $\left.\int_{z_{N}<-\delta}|z| \leq 1\right)$

$$
\int_{\substack{|z| \leq 1 \\ z_{N}>\delta}} \mu(d z) \propto \int_{\delta}^{1} \int_{B\left(0, \sqrt{1-z_{N}^{2}}\right)} \frac{d z^{\prime}}{\left|z^{\prime 2}+z_{N}^{2}\right|^{(N+\alpha) / 2}} d z_{N}=\int_{\delta}^{1}\left|z_{N}\right|^{-\alpha-1} G_{\alpha}\left(z_{N}\right) d z_{N}
$$


where $G_{\alpha}$ is the $(N-1)$-dimensional integral

$$
G_{\alpha}\left(z_{N}\right)=\int_{B\left(0, \sqrt{1 / z_{N}^{2}-1}\right)} \frac{d y}{\left(y^{2}+1\right)^{(N+\alpha) / 2}} .
$$

Now since $N+\alpha>N-1$ for any $\alpha>0, G_{\alpha}\left(z_{N}\right)$ converges as $z_{N} \rightarrow 0$ so that we obtain the first result: as $\delta \rightarrow 0$,

$$
\int_{\substack{|z| \leq 1 \\ z_{N}>\delta}} \mu(d z) \propto \int_{\delta}^{1}\left|z_{N}\right|^{-\alpha-1} d z_{N} \propto \delta^{-\alpha} .
$$

The two following estimates (on $\left\{z_{N}>\delta\right\}$ and $\left\{z_{N}<-\delta\right\}$ ) are based on the same decomposition: integrating $|z|$ leads to replace $\alpha$ with $\alpha-1$. It comes

$$
I_{\delta}^{\mathrm{int}, 2}=\int_{\delta}^{1} d z_{N} z_{N}^{-\alpha} \int_{\left|z^{\prime}\right|<\sqrt{z_{N}^{-2}-1}} \frac{d z^{\prime}}{\left(1+\left|z^{\prime}\right|^{2}\right)^{(N+\alpha) / 2}} .
$$

The second integral (with $\left|z_{N}\right|$ fixed) converges. Hence

$$
I_{\delta}^{\mathrm{int}, 2} \propto \int_{\delta}^{1} z_{N}^{-\alpha} d z_{N}
$$

and the corresponding estimate follows from this equation. The two remaining non-trivial estimates are proved similarly.

3.3. The natural assumptions on the measures and conclusion for the fractional Laplacian. Instead of plugging directly these estimates in (14), we are going to point out the main properties which allow us to conclude. While doing it, we emphasize the key assumptions needed in the general case. These assumptions reflect the fact that the measures have to be singular enough at 0 in order to avoid loss of boundary conditions. Indeed, if the measures $\left(\mu_{x}\right)_{x}$ are not singular enough, then such loss of boundary conditions may occur (cf. [7]).

A. First we concentrate on the "exterior" terms and more precisely on the first bracket in (14). We notice that for the fractional Laplacian

$$
\left|I_{\delta, r}^{\mathrm{ext}, 2}(y)\right| \leq I_{\delta, r}^{\mathrm{ext}, 3}(y) \leq O(\delta) I_{\delta, r}^{\mathrm{ext}, 1}(y) ;
$$

this will be our first key assumption. Remark that the first inequality is always true. Recalling that $\delta=o(\eta)$ and if we choose $\varepsilon \gg \eta$, the main consequence of this assumption, for the fractional Laplacian case but also in general, is the following: for $\eta$ small enough, we have

$$
\nu I_{\delta, r}^{\mathrm{ext}, 1}(y)-\frac{k_{1}+o(1)}{\eta} I_{\delta, r}^{\mathrm{ext}, 2}(y)-\frac{o(1)}{\varepsilon} I_{\delta, r}^{\mathrm{ext}, 3}(y) \geq(\nu+o(1)) I_{\delta, r}^{\mathrm{ext}, 1}(y) \geq 0 .
$$

B. On the other hand, for the "interior" terms, we first remark that, in the flat boundary case, the term $I_{\beta(\eta), r}^{\text {int, }}(y)$ is a "good" positive term. In the arguments below, there are two cases:

(i) either it is large enough to play a key role which leads to the assumption

$$
I_{\beta(\eta), r}^{\mathrm{int}, 2}(y) \rightarrow+\infty \quad, \quad I_{\beta(\eta), r}^{\mathrm{int}, 1}(y) \leq \frac{O(1)}{\beta(\eta)} I_{\beta(\eta), r}^{\mathrm{int}, 2}(y) \quad, \quad I_{\beta(\eta), r}^{\mathrm{int}, 3}(y) \leq O(1) I_{\beta(\eta), r}^{\mathrm{int}, 2}(y) ;
$$

(note that this interior term may play a key role when $\int|z| d \mu_{x}(z)=+\infty$, which corresponds to $\alpha>1$ in the fractional Laplace case). The consequence of (16) is that if we choose $\beta(\eta)$ such that $\beta(\eta)=\eta / o(1)$ and $\varepsilon(\eta)=\eta / o(1)$, then

$$
-2\|u\|_{\infty} I_{\beta(\eta), r}^{\mathrm{int}, 1}(y)+\frac{k_{1}+o(1)}{\eta} I_{\beta(\eta), r}^{\mathrm{int}, 2}(y)-\frac{o(1)}{\varepsilon} I_{\beta(\eta), r}^{\mathrm{int}, 3}(y) \geq \frac{k_{1}+o(1)}{\eta} I_{\beta(\eta), r}^{\mathrm{int}, 2}(y) .
$$


(ii) or this term is not large enough and we may assume that the interior terms are controlled by the exterior ones, namely, there exists $\beta(\eta), \varepsilon(\eta)$ such that, for $\delta \ll \eta$,

$$
I_{\beta(\eta), r}^{\mathrm{int}, 1}(y), \frac{1}{\eta} I_{\beta(\eta), r}^{\mathrm{int}, 2}(y), \frac{1}{\varepsilon(\eta)} I_{\beta(\eta), r}^{\mathrm{int}, 3}(y)=o(1) I_{\delta, r}^{\mathrm{ext}, 1}(y) .
$$

In fact, if this assumption is satisfied by $\beta(\eta)$ and $\varepsilon(\eta)$, it is also satisfied by all $\beta^{\prime}(\eta) \geq \beta(\eta)$ and $\varepsilon^{\prime}(\eta) \geq \varepsilon(\eta)$; moreover, it is natural to choose $\beta(\eta) \geq \eta$ and $\varepsilon(\eta) \gg \eta$. It is worth pointing out that we cannot choose $\beta(\eta)$ to be constant (typically $\beta(\eta)=r$ ) because of the constraints on $I_{\delta, \beta(\eta), r}^{4}(y)$ below.

The consequence of (17) is that

$$
-2\|u\|_{\infty} I_{\beta(\eta), r}^{\mathrm{int}, 1}(y)+\frac{k_{1}+o(1)}{\eta} I_{\beta(\eta), r}^{\mathrm{int}, 2}(y)+o\left(\frac{1}{\varepsilon}\right) I_{\beta(\eta), r}^{\mathrm{int}, 3}(y) \geq o(1) I_{\delta, r}^{\mathrm{ext}, 1}(y) .
$$

C. Finally, for the $I_{\delta, \beta(\eta), r}^{4}(y)$-term, we assume that

$$
I_{\delta, \beta(\eta), r}^{4}(y)=o(1) I_{\beta(\eta), r}^{\mathrm{int}, 2}(y)+o(1) I_{\delta, r}^{\mathrm{ext}, 1}(y),
$$

in order that it is controlled by either the leading interior or exterior term.

We point out that all these conditions are satisfied in the case of the fractional Laplacian. Gathering (15), (16), (17), (18) and their consequences in (14), we are just led to the (relatively) simple inequality

$$
\begin{array}{r}
\frac{k_{2}+o(1)}{\eta^{2}} a(y) D d(y) \cdot D d(y)-\frac{k_{1}+o(1)}{\eta}\left(b(y) \cdot D d(y)+\operatorname{Tr}\left(a(y) D^{2} d(y)\right)-I^{\operatorname{tr}}(y)\right) \\
+(\nu+o(1)) I_{\delta, r}^{\text {ext }, 1}(y)+\frac{k_{1}+o(1)}{\eta} I_{\beta(\eta), r}^{\text {int }, 2}(y) \leq O\left(\frac{1}{\varepsilon}\right) .
\end{array}
$$

We first recall that $\delta=o(\eta)$ and that we use $\varepsilon \gg \eta$ (which implies that the left-hand side can almost be considered as a bounded term since it grows as slowly as we want as $\eta^{-1}$ tends to $+\infty)$.

We point out that the first, third and fourth term of the left-hand side of (19) are non-negative. We next distinguish cases and use different assumptions to get the desired contradiction.

First CASE. Assume that $a(x) D d(x) \cdot D d(x)>0$. Recall that $k_{2}>0$ and that $a$ and $D d$ are continuous, it is clear that the first term (which behaves like $c / \eta^{2}$ with $c>0$ ) is enough to control the second one (which behaves like $c / \eta$ ) while the third and the fourth ones can be dropped since they are non-negative. Therefore we have a contradiction for $\eta$ small enough.

If $a(x) D d(x) \cdot D d(x)=0$, the first term cannot help in getting a contradiction and we can drop it since it is non-negative (at least for small enough $\eta$ ). We thus now work with

$$
\begin{aligned}
-\frac{k_{1}+o(1)}{\eta}(b(y) \cdot D d(y)+\operatorname{Tr}(a(y) & \left.\left.D^{2} d(y)\right)-I^{\operatorname{tr}}(y)\right) \\
& +(\nu+o(1)) I_{\delta, r}^{\mathrm{ext}, 1}(y)+\frac{k_{1}+o(1)}{\eta} I_{\beta(\eta), r}^{\mathrm{int}, 2}(y) \leq O\left(\frac{1}{\varepsilon}\right) .
\end{aligned}
$$

SECOND CASE. We examine the case when $\alpha \geq 1$. Because of Lemma 2, we have

$$
I_{\delta, r}^{\mathrm{ext}, 1}(y) \propto \delta^{-\alpha} \quad \text { and } \quad I_{\beta(\eta), r}^{\mathrm{int}, 2}(y) \propto \begin{cases}{[\beta(\eta)]^{1-\alpha}} & \text { for } \alpha>1 \\ \ln \frac{1}{\beta(\eta)} & \text { if } \alpha=1,\end{cases}
$$


both terms having the right sign and (16), (17) being both satisfied with the choice $\beta(\eta)=$ $\eta / o(1)$. Since $\delta=o(\eta)$ and $\alpha \geq 1$, the $I_{\delta, r}^{\text {ext }, 1}(y)$ is indeed the leading term because it grows faster than the $1 / \eta$ term, and we have a contradiction for $\eta$ small enough.

ThIRD CASE. We still assume that $a(x) D d(x) \cdot \operatorname{Dd}(x)=0$ but we consider the case $\alpha<1$. Taking into account the estimates of Lemma 2, the leading term is now

$$
-\frac{k_{1}+o(1)}{\eta}\left(b(y) \cdot D d(y)+\operatorname{Tr}\left(a(y) D^{2} d(y)\right)-I^{\operatorname{tr}}(y)\right)
$$

and to get a contradiction, a first sufficient condition is that

$$
b(y) \cdot D d(y)+\operatorname{Tr}\left(a(y) D^{2} d(y)\right)-I^{\operatorname{tr}}(y)<0
$$

for $r$ small enough and $y$ close to $x \in \partial \Omega$ (recall that, a priori the integral term $I^{\operatorname{tr}}(x)$ depends on $r$ ). In fact, this is equivalent to assume

$$
b(x) \cdot D d(x)+\operatorname{Tr}\left(a(x) D^{2} d(x)\right)<0
$$

since $I^{\operatorname{tr}}(y) \equiv 0$ in the fractional Laplacian case because of the symmetry of the measure. Hence, we derive a contradiction by choosing $\beta(\eta)=\eta$, remarking that the second and third term of the left-hand side of (20) have the right sign and dropping it. Since we are in the flat boundary case, we could have dropped as well the $\operatorname{Tr}\left(a(x) D^{2} d(x)\right)$ term since $D^{2} d \equiv 0$.

FOURTH CASE. The previous sufficient condition can be relaxed to

$$
b(y) \cdot D d(y)+\operatorname{Tr}\left(a(y) D^{2} d(y)-I^{\operatorname{tr}}(y) \leq 0\right.
$$

for $r$ small enough and $y$ close to $x \in \partial \Omega$ if the contradiction can be obtained from the remaining non-negative term. In the case of the fractional Laplacian, we get the contradiction for any $0<\alpha<2$ since the term $I_{\delta, r}^{\text {ext }, 1}(y)$ tends to $+\infty$ while the $I_{\beta(\eta), r}^{\text {int, }}(y)$-term has the right sign. Therefore we also obtain a contradiction in this case by choosing $\varepsilon(\eta) \gg \eta$.

Summing up the previous discussions, we conclude that there are no loss of boundary condition for for the fractional Laplacian in the flat boundary case if

$$
\begin{aligned}
\text { either } & a(x) D d(x) \cdot \operatorname{Dd}(x)>0, \\
\text { or } & \alpha \geq 1, \\
\text { or } & b(x) \cdot D d(x)+\operatorname{Tr}\left(a(x) D^{2} d(x)\right)<0, \\
\text { or } & b \cdot D d(y)+\operatorname{Tr}\left(a(x) D^{2} d(y)\right) \leq 0 \text { in a neightbourhood of } x .
\end{aligned}
$$

3.4. The general case. In this section, we consider the case of general measures $\mu_{x}$ satisfying the structure conditions (15), (16) or (17), and (18). Under these conditions, (19) holds and, as we mentioned it before, it is worth pointing out that all the terms in the left-hand side of (19) are positive, except perhaps the $b(y) \cdot D d(y)+\operatorname{Tr}\left(a(y) D^{2} d(y)\right)-I^{\operatorname{tr}}(y)$-one, which is of order $\eta^{-1}$. On the other hand, the right-hand side may grow as slowly as we wish since we can choose $\varepsilon(\eta)$.

Therefore we have two main cases: either (in a suitable sense) the term $b(y) \cdot \operatorname{Dd}(y)+$ $\operatorname{Tr}\left(a(y) D^{2} d(y)\right)-I^{\operatorname{tr}}(y)$ has the right sign or we have to control it, which means that we need another term tending to $+\infty$, faster than $\eta^{-1}$. 
This leads to the following conditions

$$
\begin{array}{ll} 
& a(x) D d(x) \cdot D d(x)>0, \\
\text { or } \quad & \int_{B}|D d(y) \cdot z| d \mu_{y}(z)=+\infty \text { uniformly w.r.t. } y \text { in a neighborhood of } x, \\
\text { or } \quad & b(y) \cdot D d(y)+\operatorname{Tr}\left(a(y) D^{2} d(y)\right)-I^{\operatorname{tr}}(y)<0 \text { in a neighborhood of } x, \\
\text { or } \quad\left\{\begin{array}{l}
b(y) \cdot D d(y)+\operatorname{Tr}\left(a(y) D^{2} d(y)\right)-I^{\operatorname{tr}}(y) \leq 0 \text { in a neighborhood of } x, \\
I_{\delta, r}^{\mathrm{ext}, 1}(y)+\frac{1}{\eta} I_{\beta(\eta), r}^{\mathrm{int}, 2}(y) \rightarrow+\infty \quad \text { as } \eta \rightarrow 0 .
\end{array}\right.
\end{array}
$$

According to the previous remarks, it is clear that the first, third and fourth conditions allow to conclude. It remains to show that the second one implies

$$
\eta I_{\delta, r}^{\mathrm{ext}, 1}(y)+I_{\beta(\eta), r}^{\mathrm{int}, 2}(y) \rightarrow+\infty \quad \text { as } \eta \rightarrow 0 .
$$

If $\int_{B}(D d(y) \cdot z)^{+} d \mu_{y}(z)=+\infty$ uniformly w.r.t. $y$, then this is true because of the $I_{\beta(\eta), r}^{\mathrm{int}, 2}(y)$ term. Otherwise we have $\int_{B}(D d(y) \cdot z)^{-} d \mu_{y}(z)=+\infty$ and using (15), this implies that $I_{\delta, r}^{\text {ext }, 1}(y)$ grows faster than $\delta^{-1}$, which allows to conclude since $\delta=o(\eta)$.

Before considering the interesting particular case of measures which are, at the same time, not very singular but symmetric, we gather in the following definition the key conditions we used in the section.

Definition 2 (Natural conditions and very singular measures). We say that the measures $\left(\mu_{y}\right)_{y}$ satisfy the condition $(N C \mu)_{x}$ for $x \in \partial \Omega$ ("natural conditions for the measures $\mu_{x}$ at $x$ ") if, for all $y$ in a neighbourhood $\mathcal{V}$ of $x$, (15), (16) or (17), and (18) holds for $\delta>d(y)$ and $\beta$

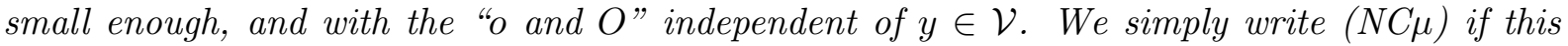
assumption is satisfied for any $x \in \partial \Omega$. if

The measures $\left(\mu_{y}\right)_{y}$ satisfy the condition $(V S \mu)_{x}$ for $x \in \partial \Omega$ ("very singular measure at $x$ ")

$$
\liminf _{y \rightarrow x} \int_{B}|D d(y) \cdot z| d \mu_{y}(z)=+\infty .
$$

This definition is used in the sequel for providing results for more general equations.

3.5. The case of weakly singular symmetric measures. In this section, we consider measures $\mu_{x}$ that are weakly singular. In the case of the fractional Laplacian, this corresponds to the case $\alpha<1$. In a general setting and freely speaking, this means that the measures have a finite first momentum (see (22) below). It turns out that in this case, the result of the technical lemma 1 can be improved to get result in the spirit of the fourth case of (21) but under weaker structure conditions on $\mu$.

Let us first explain what we call a weakly singular measure. It is a measure such that

$$
\sup _{x \in \bar{\Omega}}\left(\int_{B}|z| \mu_{x}(d z)\right)<+\infty .
$$

Under the assumption (22), the conclusion of Lemma 1 can be improved as follows.

Lemma 3. Assume (22). Then the conclusions of Lemma 1 remain true changing $b$ in $\tilde{b}=$ $b+\int z \mu_{y}(d z)$ and changing $I_{\eta, \delta, r}(y)$ in

$$
\tilde{I}_{\eta, \delta, r}(y)=-\nu I_{\delta, r}^{\mathrm{ext}, 1}(y)+2\|u\|_{\infty} I_{\beta(\eta), r}^{\mathrm{int}, 1}(y)+O\left(\frac{1}{\eta}\right) \tilde{I}_{\delta, \beta(\eta), r}^{4}(y),
$$


with

$$
\tilde{I}_{\delta, \beta, r}^{4}(y):=\int_{\mathcal{A}_{\delta, \beta, r}(y)}|z| d \mu_{y}(z)
$$

We skip the proof of this result which follows along the lines of the one of Lemma 1 . We just point out three differences: first, because of (22), we can drop the $D \psi$-terms in $I_{\delta}^{1}[\psi](\bar{x})$

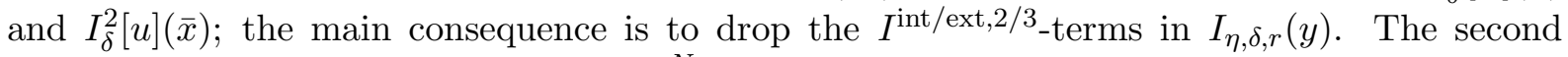
remark concerns the decomposition of $\mathbb{R}^{N}$ which is less sophisticated here. Finally, to estimate the $\tilde{I}_{\delta}^{4}(y)$-term, we do not use anymore the second derivative of $\psi$ but only an estimate of its Lipschitz constant (recall that $\varepsilon(\eta) \gg \eta$ ).

Using this result, the analogue of (14) is

$$
\begin{aligned}
\frac{k_{2}+o(1)}{\eta^{2}} a(y) D d(y) \cdot D d(y) & -\frac{k_{1}+o(1)}{\eta}\left(\tilde{b}(y) \cdot D d(y)+\operatorname{Tr}\left(a(y) D^{2} d(y)\right)\right) \\
& +\nu I_{\delta, r}^{\mathrm{ext}, 1}(y)-2\|u\|_{\infty} I_{\beta(\eta), r}^{\mathrm{int}, 1}(y) \leq O\left(\frac{1}{\eta}\right) \tilde{I}_{\delta, \beta(\eta), r}^{4}(y)+O\left(\frac{1}{\varepsilon}\right) .
\end{aligned}
$$

Structure conditions (17) and (18) of Definition 2 are replaced with (22) and: there exists $\beta(\eta)$ such that

$$
\begin{array}{cc}
I_{\beta(\eta), r}^{\mathrm{int}, 1}(y)=o(1) I_{\delta, r}^{\mathrm{ext}, 1}(y) & \text { if } \delta=o(\eta) \\
\tilde{I}_{\delta, \beta(\eta), r}^{4}(y)=o(\eta) I_{\delta, r}^{\mathrm{ext}, 1}(y) & \text { if } \delta=o(\eta)
\end{array}
$$

We point out that (15) and (16) are not relevant anymore since 2/3-integral terms disappear for weakly singular measures.

Let us mention that this result is optimal in the sense that it is proved in [7] that if the measure is finite, then a loss of boundary data actually occurs when $a=b=0$. We sum up this in the following proposition.

Proposition 1. Let us assume (22),(24) and (25). If

$$
\begin{aligned}
& a(x) D d(x) \cdot D d(x)>0, \\
& \text { or } \quad b(y) \cdot D d(y)+\operatorname{Tr}\left(a(y) D^{2} d(y)\right)-\int_{B}[D d(y) \cdot z] \mu_{y}(d z)<0 \quad \text { in a neighborhood of } x, \\
& \text { or } \quad\left\{\begin{array}{l}
b(y) \cdot D d(y)+\operatorname{Tr}\left(a(y) D^{2} d(y)\right)-\int_{B}[D d(y) \cdot z] \mu_{y}(d z) \leq 0 \quad \text { in a neighborhood of } x \text { and } \\
I_{\beta(\eta), r}^{\mathrm{ext}, 1}(y) \rightarrow+\infty \quad \text { as } \eta \rightarrow 0,
\end{array}\right.
\end{aligned}
$$

then any solution $u$ of (7)-(2) takes the boundary data in the classical sense at $x$.

In particular, this result applies to the case of fractional Laplacian operators of order $0<\alpha<$ 1 , and even to the limiting case $\alpha=0$, so-called "zero-Laplacian". Let us notice that in this case (as well as for any centered measure), the $\int_{B}[D d(y) \cdot z] \mu_{y}(d z)$-term vanishes.

Remark 4. We point out that obviously, if one is only interested in looking at the boundary condition at a single point $x=x_{0}$, the structure conditions on the singular measure $(22),(24)$ and (25) are only requested to hold locally uniformly for $y$ near $x_{0}$. For instance, one could think of a measure which is very singular at some points $x$ and only weakly singular at some other points. 


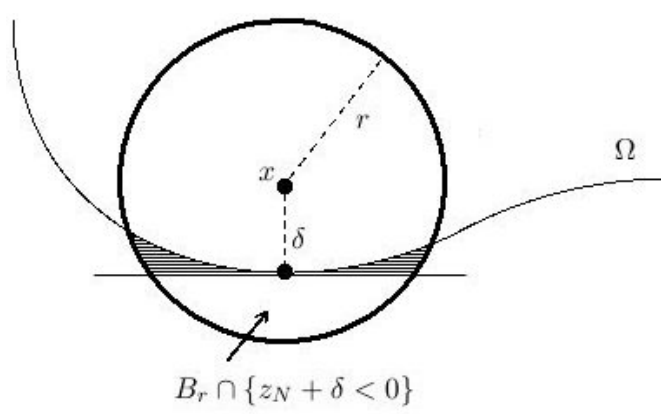

Figure 2. Reduction to the flat case

3.6. Dirichlet problem in a domain. We would like to be able to get sufficient conditions ensuring that the Dirichlet boundary condition is satisfied in the classical sense in the case of a general smooth domain too. In view of the previous discussion, we see that it is enough to ensure that the measures $\mu_{x}$ still satisfy the conditions in section 3.3. Condition (27) below shows that under some rather natural condition on the measures $\mu_{x}$, one can pass from a half-space type domain to a general $C^{2}$-smooth domain: for any $\gamma>0$ small enough, for any $c>0$ and for any $\mathbf{e} \in \mathbb{R}^{N},|\mathbf{e}|=1$,

$$
\left\{\begin{array}{l}
\mu_{x}\left\{z: \gamma<\mathbf{e} \cdot z<\gamma+c|z|^{2}\right\}=o_{\gamma}(1) \cdot \mu_{x}\{z: \mathbf{e} \cdot z>\gamma\} \\
\mu_{x}\left\{z:-\gamma-c|z|^{2}<\mathbf{e} \cdot z<-\gamma\right\}=o_{\gamma}(1) \cdot \mu_{x}\{z: \mathbf{e} \cdot z<-\gamma\}
\end{array}\right.
$$

where $o_{\gamma}(1)$ is uniform wrt $x$ (see Figure 2: the hatched region should be neglectable in front of the grey one).

Indeed, thanks to these assumptions, it appears that the integrals $I^{\text {int/ext,1/3 }}$ in the case of a $C^{2}$-domain are comparable to the same integrals in the flat case. Now the difference between the integrals $I^{\mathrm{int} / \mathrm{ext}, 2}$ in the general and flat case are neglectable in front of $I^{\mathrm{int} / \mathrm{ext}, 3}$ (non flat case), which is equivalent to say that this is also neglectable in front of $I^{\text {int/ext,3 }}$ (flat case). Thus, if the main assumptions on the measure is satified in the flat case, under hypothesis (27) they are also satisfied in the general case of a $C^{2}$-domain.

To illustrate this, let us prove that property (27) holds in the case of the fractional Laplace operator, $\mu(z)=1 /|z|^{N+\alpha}$ with $0 \leq \alpha<2$. Let us first treat the case $\alpha \in(0,2)$, and let $\mathbf{e}$ be a unit vector of $\mathbb{R}^{N}$. By Lemma 2 , we know that the integral of $\mu$ over $B_{r} \cap\{\mathbf{e} \cdot z>\gamma\}$ is of order $\gamma^{-\alpha}$, so it is sufficient to prove that $\gamma^{\alpha} \Lambda(\gamma)=o(1)$ with

$$
\Lambda(\gamma)=\int_{B_{r} \cap\left\{0<\mathbf{e} \cdot z-\gamma<c|\tilde{z}|^{2}\right\}} \frac{d z}{|z|^{N+\alpha}} .
$$

Letting $z_{N}$ denote $(\mathbf{e} \cdot z)$ and $z^{\prime}=z-z_{N} \mathbf{e}$, we first write

$$
\Lambda(\gamma)=\int_{\left|z^{\prime}\right|<r} \int_{\gamma}^{\gamma+c\left|z^{\prime}\right|^{2}} \frac{d z_{N}}{\left(z^{\prime 2}+z_{N}^{2}\right)^{(N+\alpha) / 2}} d z^{\prime}=\int_{\left|z^{\prime}\right|<r}\left|z^{\prime}\right|^{1-N-\alpha} G_{\alpha}\left(\gamma, z^{\prime}\right) d z^{\prime}
$$

where

$$
G_{\alpha}\left(\gamma, z^{\prime}\right)=\int_{\gamma /\left|z^{\prime}\right|}^{\gamma /\left|z^{\prime}\right|+c\left|z^{\prime}\right|} \frac{d t}{\left(1+t^{2}\right)^{(N+\alpha) / 2}}
$$

Now we can estimate $\Lambda(\gamma)$ as follows:

$$
\Lambda(\gamma)=\int_{\left|z^{\prime}\right| \leq \gamma^{\beta}}\{\ldots\}+\int_{\gamma^{\beta} \leq\left|z^{\prime}\right| \leq r}\{\ldots\}
$$


for $\beta>0$ to be chosen later. Now consider $z^{\prime}$ such that $\left|z^{\prime}\right| \leq \gamma^{\beta}$. If one chooses $\beta>1$, then $\frac{\gamma}{\left|z^{\prime}\right|} \gg 1$ and $c\left|z^{\prime}\right| \ll 1$. Hence we get for such $z^{\prime}$

$$
\begin{aligned}
G_{\alpha}\left(\gamma, z^{\prime}\right) & \leqslant \int_{\gamma /\left|z^{\prime}\right|}^{\gamma /\left|z^{\prime}\right|+c\left|z^{\prime}\right|} \frac{d t}{t^{N+\alpha}} \\
& \leqslant c\left|z^{\prime}\right| \times\left(\frac{\gamma}{2\left|z^{\prime}\right|}\right)^{-N-\alpha} \\
& \leqslant C \gamma^{-N-\alpha}\left|z^{\prime}\right|^{N+\alpha+1} .
\end{aligned}
$$

On the other hand for $\left|z^{\prime}\right|>\gamma^{\beta}$, since $\left(1+t^{2}\right)^{(N+\alpha) / 2} \geqslant 1$,

$$
G_{\alpha}\left(\gamma, z^{\prime}\right) \leq 2 c\left|z^{\prime}\right|
$$

Thus we have finally (remember that the integrals on $z^{\prime}$ are $(N-1)$-dimensional) for $\alpha \neq 1$

$$
\begin{aligned}
\Lambda(\gamma) & \leq C\left(\int_{\left|z^{\prime}\right|<\gamma^{\beta}}\left|z^{\prime}\right|^{2} \gamma^{-N-\alpha} d z^{\prime}+\int_{\gamma^{\beta}<\left|z^{\prime}\right|<r}\left|z^{\prime}\right|^{2-N-\alpha} d z^{\prime}\right) \\
& \leq C\left(\gamma^{-N-\alpha} \int_{0}^{\gamma^{\beta}} t^{N} d t+\int_{\gamma^{\beta}}^{r} t^{-\alpha} d t\right) \\
& \leq C \gamma^{-\alpha}\left(\gamma^{(N+1) \beta-N}+\gamma^{\beta(1-\alpha)+\alpha}\right) .
\end{aligned}
$$

Since $\beta>1$, we have $(N+1) \beta>N$ so the first term is good. If $\alpha \in[0,1)$, then $\beta(1-\alpha)+\alpha>0$ and the second term is still good. If $\alpha>1$, we choose $\beta \in\left(1, \frac{\alpha}{\alpha-1}\right)$. Now if $\alpha=1$, we have

$$
\gamma \Lambda(\gamma) \leq C\left(\gamma^{(N+1) \beta-N}+\gamma \ln \frac{1}{\gamma}\right)
$$

and we conclude in the same way (even more easily).

Finally in the case $\alpha=0$, we get the same estimate (with $\alpha=0$ ) which proves that $\Lambda(\gamma)$ remains bounded as $\gamma \rightarrow 0$, for any choice of $\beta>0$. Then since the mass of $\mu$ in $B_{r} \cap\{z: \mathbf{e} \cdot z>\gamma\}$ is of order $\ln (1 / \gamma)$, we obtain that $\ln (1 / \gamma)^{-1} \Lambda(\gamma)=o(1)$. (27).

Thus we conclude that for any $0 \leqslant \alpha<2$, the measure $d \mu=d z /|z|^{N+\alpha}$ satisfies the property

3.7. General results in the case of a linear equation. We discussed thoroughly the model linear equation (7) and we would like to state the result we finally proved. We only state it for fractional Laplace operators. Since we also want to include the existence and uniqueness results provided by Theorem 1 , we introduce the following conditions on $a, b$ and $f$, which ensure, in particular, that (A3-1) holds.

(HD) $a=\sigma \sigma^{T}$ with $\sigma$ bounded and Lipschitz continuous on $\bar{\Omega}, b$ is bounded and Lipschitz continuous on $\bar{\Omega}$ and $f$ is bounded and continuous on $\bar{\Omega}$.

We formulate the following result in two parts: in the first one, we provide conditions under which there is no loss of boundary conditions using minimal hypotheses on $a, b$ and $f$, while, in the second one, we give a general existence and uniqueness result under more restrictive assumptions on $a, b$ and $f$, namely (HD).

Theorem 2. Assume that $a, b, f$ are continuous functions defined on $\bar{\Omega}$, where $\Omega$ is a $C^{2}$ domain, $a(x)$ being a symmetric non-negative matrix for any $x \in \bar{\Omega}, d \mu_{x}(z)=d \mu(z)=$ 
$d z /|z|^{N+\alpha}(0 \leq \alpha<2)$ and that $g$ is bounded and continuous on $\Omega^{c}$. If, at a given point $x \in \partial \Omega$,

$$
\left\{\begin{aligned}
\text { either } & a(x) D d(x) \cdot D d(x)>0, \\
\text { or } & \alpha \geq 1, \\
\text { or } & b(y) \cdot D d(y)+\operatorname{Tr}\left(a(y) D^{2} d(y)\right) \leq 0 \quad \text { for } y \text { in a neighborhood of } x,
\end{aligned}\right.
$$

then any usc subsolution $u$ of the Dirichlet problem (7)-(2) (resp. any lsc supersolution $v$ ), satisfies $u(x) \leq g(x)$ (resp. $v(x) \geq g(x))$.

If these conditions hold for any $x \in \partial \Omega$ and if (HD) holds, there then exists a unique continuous solution of the Dirichlet problem which assumes the boundary condition in the classical sense.

Remark 5. We point out that Condition (28) is (almost) exactly (21): indeed, $I_{r}^{\operatorname{tr}}(x)=0$ for any $x$ (since the measure is radially symmetric) and the second condition of (21) is equivalent to $\alpha \geq 1$. However we wrote $b(y) \cdot D d(y)+\operatorname{Tr}\left(a(y) D^{2} d(y)\right) \leq 0$ in a neighborhood of $x$ instead of the stronger condition $b(x) \cdot D d(x)+\operatorname{Tr}\left(a(x) D^{2} d(x)\right)<0$ to take into account the case when the equation reduces to the non-local term, for instance when $0 \leq \alpha<1$ and $a=b=0$.

3.8. More general operators. We would like to conclude this section by exhibiting other measures that can be handled with the techniques we presented above to deal with the linear equation. For instance, in mathematical finance [17], tempered $\alpha$-stable Lévy measures in one dimensional space appear in several models. They can be written under the following form

$$
\mu(d z)=c_{+} \frac{e^{-\gamma_{+} z}}{|z|^{1+\alpha}} \mathbf{1}_{(0,+\infty)}(z) d z+c_{-} \frac{e^{\gamma_{-} z}}{|z|^{1+\alpha}} \mathbf{1}_{(-\infty, 0)}(z) d z .
$$

One can check that such a measure satisfies the conditions A,B and $\mathrm{C}$ of section 3.3 and hence the previous results apply.

To finish with, let us briefly explain how to handle Lévy-Itô operators of the form (5) by considering proper $x$-dependent measures. Assume for simplicity that $\mu=\frac{d z}{|z|^{N+\alpha}}$. We first need to assume that there exists an inverse map for $j(x, z)$ that has some continuity properties. Precisely assume that there exists $J(x, Z)$ such that

$$
j(x, J(x, Z))=Z \quad J(x, j(x, z))=z .
$$

Then the operator (5) can be rewritten as

$$
\mathcal{I}_{L I}[u](x)=\int\left(u(x+Z)-u(x)-\nabla u(x) \cdot Z \mathbf{1}_{B}(J(x, Z))\right) \frac{\left|\operatorname{det} D_{Z} J(x, Z)\right|}{|J(x, Z)|^{N+\alpha}} d Z .
$$

If one can ensure next that there exists two constants $c_{0}, C_{0}$ such that

$$
c_{0}|Z| \leq|J(x, Z)| \leq C_{0}|Z|,
$$

then we end up with a measure

$$
\mu_{x}(d Z)=\frac{\left|\operatorname{det} D_{Z} J(x, Z)\right|}{|J(x, Z)|^{N+\alpha}} d Z \leq C_{0}^{-N-\alpha} \frac{\left|\operatorname{det} D_{Z} J(x, Z)\right|}{|Z|^{N+\alpha}} d Z
$$

assuming that $x \mapsto D_{Z} J(x, Z)$ is continuous is now enough to conclude. 


\section{Two NON-Linear EXamples AND the PARABOlic CASE}

We present in this section a few examples of applications of our techniques to non-linear equations. We also briefly discuss the parabolic case.

Following the explicit computations of Section 3, we keep working with the fractional Laplace operator and we choose $\delta=d(y)$ in Lemma 1. In particular the measure $\mu$ satisfies conditions $\mathrm{A}, \mathrm{B}$ and $\mathrm{C}$ of section 3.3 where integral terms are defined in (9).

4.1. Hamilton-Jacobi-Bellman Equations. Next we turn to the case of Hamilton-JacobiBellman Equations, namely

$$
\sup _{\beta \in \mathcal{B}}\left\{-\operatorname{Tr}\left(a^{\beta}(x) D^{2} u\right)-b^{\beta}(x) \cdot D u-\mathcal{I}^{\beta}[u](x)+u-f^{\beta}(x)\right\}=0 \quad \text { in } \Omega,
$$

where $\mathcal{B}$ is a compact metric space, $a^{\beta}, b^{\beta}, f^{\beta}$ are continuous functions and $\mathcal{I}^{\beta}$ are non-local Lévy-Itô operators associated with $\mu^{\beta}, j^{\beta}$ satisfying (4) and (6) with $\bar{c}>0$ being independent of $\beta$. We assume also the following "equi-integrability property":

There exists $\mu, j$ satisfying (A1) such that $\left|j^{\beta}(x, z)\right| \leq|j(x, z)|$ for any $x, z$ and $\mu^{\beta} \leq \mu$ in the sense that for any $\mu$-integrable, non-negative function $g$,

$$
\int_{\mathbb{R}^{N}} g(z) d \mu^{\beta}(z) \leq \int_{\mathbb{R}^{N}} g(z) d \mu(z)
$$

All the above "basic" conditions on $\mathcal{I}^{\beta}$ will be referred to below as (IP) for "integrability property".

This equation does not enter into the frameworks of Theorem 1 and Lemma 1 since we deal with several non-local terms at the same time. But these results extend to this more general case, under suitable assumptions, since the methods to prove them readily apply: indeed, the above Hamilton-Jacobi-Bellman equation is a supremum of a family of linear operators and we can treat it in a similar way as the case of linear equations if assumptions are uniform enough wrt $\beta$; but, of course, the supremum creates also some dissymmetry.

To be more precise, we introduce the following condition, which again ensures that a (A3-1)type assumption holds.

(HD-HJB) $a^{\beta}, b^{\beta}$ satisfy (HD) with $L^{\infty}$-bounds and Lipschitz constants being independent of $\beta, f^{\beta}$ is uniformly bounded in $\bar{\Omega}$ and uniformly continuous in compact subsets of $\mathbb{R}^{N}$, uniformly wrt $\beta$. For any $\beta \in \mathcal{B}, \mathcal{I}^{\beta}$ satisfy (A1) with a constant $\bar{c}$ which is independent of $\beta$.

Our result is the

Theorem 3. Assume that $\Omega$ is a $C^{2}$-domain, $a^{\beta}, b^{\beta}$, $f^{\beta}$ are continuous functions defined on $\bar{\Omega}, g$ is bounded and continuous on $\Omega^{c}$ and that the non-local terms $\mathcal{I}^{\beta}$ satisfy (IP).

If, at $x \in \partial \Omega$, there exists $\beta \in \mathcal{B}$ such that $\mu^{\beta}$ satisfies $(N C \mu)_{x}$ (see Definition 2) and if one of the following conditions holds

(i) $a^{\beta}(x) \operatorname{Dd}(x) \cdot \operatorname{Dd}(x)>0$,

(ii) $\mu^{\beta}$ satisfies $(V S \mu)_{x}$ (see Definition 2),

(iii) $b^{\beta}(x) \cdot D d(x)+\operatorname{Tr}\left(a^{\beta}(x) D^{2} d(x)\right)-\liminf _{\substack{x \rightarrow 0 \\ y \rightarrow x}} I^{\beta, \operatorname{tr}}(y)<0$,

then any usc subsolution $u$ of the Dirichlet problem (7)-(2) satisfies $u(x) \leq g(x)$.

If, at $x \in \partial \Omega$, there exists $\theta>0$ and $\mathcal{B}_{1}(x), \mathcal{B}_{2}(x), \mathcal{B}_{3}(x)$ such that $\mathcal{B}=\mathcal{B}_{1}(x) \cup \mathcal{B}_{2}(x) \cup \mathcal{B}_{3}(x)$ with 
(i)' for all $\beta \in \mathcal{B}_{1}(x), a^{\beta}(x) D d(x) \cdot D d(x)>\theta$,

(ii)' for all $\beta \in \mathcal{B}_{2}(x)$, Condition $(N C \mu)_{x}$ of Definition 2 hold for any $\mu^{\beta}$, uniformly w.r.t $\beta$

(iii)' for all $\beta \in \mathcal{B}_{3}(x), b^{\beta}(x) \cdot D d(x)+\operatorname{Tr}\left(a^{\beta}(x) D^{2} d(x)\right)-\liminf _{\substack{x \rightarrow 0 \\ y \rightarrow x}} I_{r}^{\beta, \operatorname{tr}}(y)<-\theta$,

then any lsc supersolution $v$ of the Dirichlet problem (7)-(2) satisfies $v(x) \geq g(x)$.

If these two conditions hold for any $x \in \partial \Omega$, and if (HD-HJB) holds, then there exists a unique continuous solution of the Dirichlet problem which assumes the boundary condition in the classical sense.

4.2. Fractional Laplace Equations. Our last stationary example is concerned with the case of fractional Laplace equations involving a non-linearity in the gradient

$$
(-\Delta)^{\alpha / 2} u-b(x) \cdot D u+c(x)|D u|^{m}+u=f(x) \text { in } \Omega,
$$

where $0<\alpha<2, b, c$ are continuous functions, $c \geq 0$ on $\bar{\Omega}$ and $m>0$.

By Section 3, we know how the fractional Laplacian term behaves (see Subsection 3.2). The main interest of this example is to see the interaction of this term with the non-linear term $c(x)|D u|^{m}$ and try to figure out what are the "allowed" powers $m$.

Since the term $c(x)|D u|^{m}$ is non-negative, the most interesting analysis of loss of boundary condition concern supersolutions. The fractional Laplacian term provides a $\delta^{-\alpha}$ contribution while the linear $b$-term yields $k_{1} \eta^{-1}$ and the $c$-term yields $c(x) k_{1}^{m} \eta^{-m}$. As above the $\delta^{-\alpha}$ contribution dominates if $\alpha \geq 1$ and if $m \leq \alpha$ (recall that $\delta=o(1) \eta$ ). If $0<\alpha<1$, then the linear $b$ term may play the main role (see Section 5 ) and, if it is the case, then $m<1$ is the natural condition.

We can formulate the

Theorem 4. Assume that $b, c, f$ are continuous functions, that $\Omega$ is a $C^{2}$-domain and $g$ is bounded and continuous on $\Omega^{c}$. If, at $x \in \partial \Omega$, we have one of the following assumptions

(i) $\alpha \geq 1$

(ii) $b(y) \cdot D d(y) \leq 0$ in a neighborhood of $x$

then any usc subsolution $u$ of the Dirichlet problem (30)-(2) satisfies $u(x) \leq g(x)$.

If, at $x \in \partial \Omega$, we have one of the following assumptions

(i)' $\alpha \geq 1$ and $m \leq \alpha$

(ii)' $b(x) \cdot D d(x) \leq 0$ and $m<1$

(iii)' $\alpha \geq 0, m \leq \alpha$ and $b(y) \cdot D d(y) \leq 0$ in a neighborhood of $x$

then any lsc supersolution $v$ of the Dirichlet problem (30)-(2) satisfies $v(x) \geq g(x)$.

If these two sets of conditions hold for any $x \in \partial \Omega$ and if $b, c$ are Lipschitz continuous then there exists a unique continuous solution of the Dirichlet problem which assumes the boundary condition in the classical sense.

4.3. Parabolic Equations. We conclude this section by remarking that, as far as losses of boundary conditions are concerned ( $c f$. Lemma 1), the parabolic case can be treated exactly in the same way and leads to similar conditions. Indeed, if one considers a problem set in $\mathcal{O} \times(0, T)$, the parabolic pde can be seen as a degenerate elliptic pde set in $\Omega=\mathcal{O} \times(0, T)$. The domain $\Omega$ is $C^{2}$ if $\mathcal{O}$ is $C^{2}$ and the non-linearity $F$ is replaced with

$$
\tilde{F}\left(x, t, u,\left(p_{t}, p_{x}\right), X\right)=p_{t}+F\left(x, t, u, p_{x}, X\right),
$$


where $p_{t}$ and $p_{x}$ correspond to the derivatives wrt the time and space variables respectively. In the parabolic case, Lemma 1 readily applies by using the domain $\Omega$ and the non-linearity $\tilde{F}$. We point out that the signed distance function to the boundary of $\Omega$ coincides with the signed distance function to the boundary of $\mathcal{O}$ and therefore the statement of Lemma 1 is essentially the same except that one has to: (i) add the extra time variable and (ii) replace $F$ in equations (10) and (11) with $o\left(\eta^{-1}\right)+F$, the $o\left(\eta^{-1}\right)$ coming from the $u_{t}$ (or $p_{t}$ ) term.

\section{Influence of the Drift Term}

In this section we show that under some circumstances, the boundary data may be lost, even if the measure is singular and not integrable near the origin. This phenomenon happens due to the fact that the influence of the drift term may be greater than that of the non-local term. In the case of the fractional Laplace operator, this may happen when $\mu(z)=1 /|z|^{N+\alpha}$ with $0<\alpha<1$ (for $1 \leq \alpha<2$ on the contrary, the boundary data is always taken even if we have a drift term).

We shall construct here an example of such a situation in dimension $N=1$, for a measure $\mu$ similar to the fractional Laplace:

$$
\mu(z)=\frac{1}{|z|^{1+\alpha}} \mathbf{1}_{B}
$$

Considering the following model equation

$$
\mathcal{L} u:=-\int\{u(x+z)-u(x)\} \mu(d z)-u_{x}=f(x) \text { in } \mathbb{R}_{+},
$$

we have the

Proposition 2. For any $0 \leq \alpha<1$, there exists a bounded continuous function $f_{\alpha}: \mathbb{R}_{+} \rightarrow \mathbb{R}$ and a solution $u=u_{\alpha}$ of $\mathcal{L}(u)=f_{\alpha}$ in $\{x \geq 0\}$, with $u=0$ in $\{x<0\}$ such that $u(0)=1>0$.

Proof. Let us consider $k$ exponents $\gamma_{i} \in(0,1), i=1, \ldots, k$ where $k \geq 1$ is an integer to be fixed therafter, satisfying $\gamma_{i+1}<\gamma_{i}$, and a function $u$ as follows

$$
u(x)=a_{1} x^{\gamma_{1}}+a_{2} x^{\gamma_{2}}+\cdots+a_{k} x^{\gamma_{k}}+1 \text { for } x \geq 0,
$$

and $u=0$ for $x<0$. We shall compute the operator applied to $u$ and thus obtain a bounded function $f(x)$ for a suitable choice of the parameters $\left(\gamma_{i}\right)_{i}$.

For any $\gamma_{i}>0$ and $0 \leq x<1$, we claim that

$$
\mathcal{L}\left(x^{\gamma_{i}}\right)=l_{i} x^{\gamma_{i}-\alpha}+M_{i}(x)-\gamma_{i} x^{\gamma_{i}-1}
$$

where $l_{i}$ is a constant and $M_{i}(x)$ is a continuous function. Now we gather terms and obtain

$$
\begin{aligned}
\mathcal{L} u(x)= & \sum_{i=1}^{k-1}\left(a_{i} l_{i} x^{\gamma_{i}-\alpha}-a_{i+1} \gamma_{i+1} x^{\gamma_{i+1}-1}\right)+a_{k} l_{k} x^{\gamma_{k}-\alpha}+\left(l_{0} x^{-\alpha}-a_{1} \gamma_{1} x^{\gamma_{1}-1}\right) \\
& +\sum_{i=0}^{k} a_{i} M_{i}(x) .
\end{aligned}
$$

We next choose

$$
\gamma_{i}=i(1-\alpha) \quad \text { and } \quad a_{1}=\frac{l_{0}}{\gamma_{1}} \quad \text { and } \quad a_{i+1}=\frac{a_{i} l_{i}}{\gamma_{i+1}}
$$


so that, in particular, $\gamma_{i}-\alpha=\gamma_{i+1}-1$ and $-\alpha=\gamma_{1}-1$, and we obtain

$$
\mathcal{L} u(x)=a_{k} l_{k} x^{\gamma_{k}-\alpha}+\sum_{i=0}^{k} a_{i} M_{i}(x) .
$$

To finish with, choose $k$ large enough such that $\gamma_{k} \geq \alpha$; precisely, $k \geq \frac{\alpha}{1-\alpha}$. Eventually, we arrive at a bounded right-hand side

$$
f(x)=\mathcal{L}\left(a_{1} x^{\gamma_{1}}+a_{2} x^{\gamma_{2}}+\cdots+a_{k} x^{\gamma_{k}}+1\right),
$$

which implies that we have a solution which does not take the boundary data although $f$ is bounded and continuous up to the boundary.

\section{Remarks:}

- Note that if $\alpha$ is very small, that is, if $0 \leq \alpha \leq 1 / 2$, then the condition $k \geq \alpha /(1-\alpha)$ is fulfilled for $k=1$. Hence we obtain a counter-example of the form $u(x)=1+a \cdot x^{1-\alpha}$.

- In the case of the zero-Laplacian, the counter-example has the form $u(x)=1+a \cdot x$, that is, we have an affine function.

- As $\alpha \rightarrow 1^{-}, k$ increases and we cannot construct a counter-example when $\alpha=1$ which would require an infinite number of powers. Indeed, we know that when the measure is singular enough, the boundary data is always taken, even if a drift term is involved in the equation. 


\section{REFERENCES}

[1] O. Alvarez and A. Tourin. Viscosity solutions of non-linear integro-differential equations. Ann. Inst. H. Poincaré Anal. Non Linéaire 13 (3) (1996), 293-317.

[2] M. Arisawa. A new definition of viscosity solutions for a class of second-order degenerate elliptic integrodifferential equations. Ann. I. H. Poincaré, 23(5):695-711, 2006.

[3] G. Barles and J. Burdeau. The Dirichlet problem for semilinear second-order degenerate elliptic equations and applications to stochastic exit time control problems. Comm. in PDE 20 (1\& 2), 1995, pp 129-178.

[4] G. Barles and C. Imbert. Second-order elliptic integro-differential equations: Viscosity solutions' theory revisited. Ann. Inst. H. Poincaré Anal. Non Linéaire, 2007. accepted for publication in 2007.

[5] I. Bensaoud and A. Sayah. Stability results for Hamilton-Jacobi equations with integro-differential terms and discontinuous Hamiltonians. Arch. Math. (Basel) 79 (2002), no. 5, 392-395.

[6] J.-M. Bony, P. Courrège and P. Priouret. Semi-groupes de Feller sur une variété à bord compacte et probléme aux limites intégro-différentiels du second ordre donnant lieu au principe du maximum. Ann. Inst. Fourier (Grenoble) 18 (1968), 369-521.

[7] E. Chasseigne. The Dirichlet problem for some non-local diffusion equations. Diff. Int. Eq. 20 (2007) 1389-1404.

[8] R. Cont and E. Voltchkova. Integro-differential equations for option prices in exponential Lévy models. Finance Stoch. 9 (2005), no. 3, 299-325.

[9] M.G Crandall, H.Ishii and P.L Lions. User's guide to viscosity solutions of second order partial differential equations. Bull. Amer. Soc. 27 (1992), pp 1-67.

[10] F. Da Lio. Strong Comparison Results for Quasilinear Equations in Annular Domains and Applications. Comm. in PDE 27 (1\& 2), 283-323 (2002).

[11] M. G. Garroni and J. L. Menaldi. Second order elliptic integro-differential problems, volume 430 of Chapman 83 Hall/CRC Research Notes in Mathematics. Chapman \& Hall/CRC, Boca Raton, FL, 2002.

[12] D. Gilbarg and N. Trudinger. Elliptic partial differential equations of second order. Second edition. Grundlehren der Mathematischen Wissenschaften [Fundamental Principles of Mathematical Sciences], 224. Springer-Verlag, Berlin, 1983.

[13] C. Imbert. A non-local regularization of first order Hamilton-Jacobi equations Journal of Differential Equations 211 (1) (2005), 214-246

[14] H. Ishii. Perron's method for Hamilton-Jacobi Equations. Duke Math. J. 55 (1987), 369-384.

[15] E. R. Jakobsen and K. H. Karlsen. A "maximum principle for semicontinuous functions" applicable to integro-partial differential equations. NoDEA Nonlinear Differential Equations Appl. .13 (2), 137-165 (2006).

[16] H. Pham. Optimal stopping of controlled jump diffusion processes: a viscosity solution approach. J. Math. Systems Estim. Control 8 (1), 27 pp. (electronic) (1998).

[17] B. Øksendal and A. Sulem. Applied stochastic control of jump diffusions. Universitext. Springer-Verlag, Berlin, 2005.

[18] A. Sayah. Équations d'Hamilton-Jacobi du premier ordre avec termes intégro-différentiels. I. Unicité des solutions de viscosité. (French) [First-order Hamilton-Jacobi equations with integro-differential terms. I. Uniqueness of the viscosity solutions] Comm. Partial Differential Equations 16 (1991), no. 6-7, 1057-1074.

[19] A. Sayah. Équations d'Hamilton-Jacobi du premier ordre avec termes intégro-différentiels. II. Existence de solutions de viscosité. (French) [First-order Hamilton-Jacobi equations with integro-differential terms. II. Existence of viscosity solutions] Comm. Partial Differential Equations 16 (1991), no. 6-7, 1075-1093.

Laboratoire de Mathématiques et Physique Théorique CNRS UMR 6083, Fédération Denis Poisson, Université François Rabelais, Parc de Grandmont, 37200 Tours, France

Laboratoire de Mathématiques et Physique Théorique CNRS UMR 6083, Fédération Denis Poisson, Université François Rabelais, Parc de Grandmont, 37200 Tours, France

Ceremade, Université Paris-Dauphine, Place de Lattre de Tassigny 75775 Paris Cedex 16, France 